\title{
Earthquake Slip Between Dissimilar Poroelastic Materials
}

\section{Citation}

Dunham, Eric M. and James R. Rice. 2008. Earthquake slip between dissimilar poroelastic materials. Journal of Geophysical Research 113(B09304). http:// dx.doi.org/10.1029/2007JB005405

\section{Published Version}

http://dx.doi.org/10.1029/2007JB005405

\section{Permanent link}

http://nrs.harvard.edu/urn-3:HUL.InstRepos:2645465

\section{Terms of Use}

This article was downloaded from Harvard University's DASH repository, and is made available under the terms and conditions applicable to Other Posted Material, as set forth at http:// nrs.harvard.edu/urn-3:HUL.InstRepos:dash.current.terms-of-use\#LAA

\section{Share Your Story}

The Harvard community has made this article openly available.

Please share how this access benefits you. Submit a story.

Accessibility 


\title{
Earthquake slip between dissimilar poroelastic materials
}

\author{
Eric M. Dunham ${ }^{1}$ and James R. Rice ${ }^{1}$ \\ Received 26 September 2007; revised 8 June 2008; accepted 18 June 2008; published 10 September 2008.
}

[1] A mismatch of elastic properties across a fault induces normal stress changes during spatially nonuniform in-plane slip. Recently, Rudnicki and Rice showed that similar effects follow from a mismatch of poroelastic properties (e.g., permeability) within fluid-saturated fringes of damaged material along the fault walls; in this case, it is pore pressure on the slip plane and hence effective normal stress that is altered during slip. The sign of both changes can be either positive or negative, and they need not agree. Both signs reverse when rupture propagates in the opposite direction. When both elastic and poroelastic properties are discontinuous across the fault, steady sliding at a constant friction coefficient, $f$, is unstable for arbitrarily small $f$ if the elastic mismatch permits the existence of a generalized Rayleigh wave. Spontaneous earthquake rupture simulations on regularized slip-weakening faults confirm that the two effects have comparable magnitudes and that the sign of the effective normal stress change cannot always be predicted solely from the contrast in elastic properties across the fault. For opposing effects, the sign of effective normal stress change reverses from that predicted by the poroelastic mismatch to that predicted by the elastic mismatch as the rupture accelerates, provided that the wave speed contrast exceeds about 5-10\% (the precise value depends on the poroelastic contrast and Skempton's coefficient). For faults separating more elastically similar materials, there exists a minimum poroelastic contrast above which the poroelastic effect always determines the sign of the effective normal stress change, no matter the rupture speed.

Citation: Dunham, E. M., and J. R. Rice (2008), Earthquake slip between dissimilar poroelastic materials, J. Geophys. Res., 113, B09304, doi:10.1029/2007JB005405.

\section{Introduction}

[2] Current understanding of the strength of faults rests on the principle of effective stress,

$$
\tau=f(\sigma-p)=f \bar{\sigma},
$$

which states that the shear strength, $\tau$, is given by the product of the coefficient of friction, $f$, and the effective normal stress, $\bar{\sigma}$. The latter may be altered by changes in normal stress, $\sigma$, and/or pore pressure, $p$. We adopt the convention that tensor stress components like $\sigma_{11}$ and $\sigma_{22}$ are positive in tension, but take $\sigma$ and $\bar{\sigma}$ as positive in compression (i.e., $\sigma=-\sigma_{22}$ for a fault with unit normal in the $x_{2}$ direction).

[3] While traditional earthquake models appealed to the reduction of $f$ from static to dynamic values [Brace and Byerlee, 1966], it has since been suggested that a reduction in $\sigma$ during spatially nonuniform slip between dissimilar elastic solids might also be responsible for dynamic weakening of faults during earthquakes [Weertman, 1980]. The

\footnotetext{
${ }^{1}$ Department of Earth and Planetary Sciences and School of Engineering and Applied Sciences, Harvard University, Cambridge, Massachusetts, USA.

Copyright 2008 by the American Geophysical Union. 0148-0227/08/2007JB005405\$09.00
}

issue has generated considerable debate recently; some authors argue that bimaterial weakening effects are important during earthquakes [Ben-Zion, 2006a, 2006b], while others claim they are not [Andrews and Harris, 2005; Harris and Day, 2005]. Despite a lack of consensus on whether or not the effect is important in earthquakes, it is well established theoretically that Coulomb frictional sliding (with constant $f$ ) between dissimilar elastic solids is unstable for a wide range of material pairs and friction coefficients, in particular those characterizing natural faults [Renardy, 1992; Adams, 1995; Ranjith and Rice, 2001].

[4] When the across-fault shear wave speed contrast is less than about $30 \%$ (contrasts in density and Poisson's ratio are less important), an interfacial wave known as the generalized Rayleigh wave exists for frictionless contact and no opening [Weertman, 1963; Achenbach and Epstein, 1967]. This level of material contrast is generally what occurs across faults [Feng and McEvilly, 1983; EberhartPhillips and Michael, 1998; Tanimoto and Sheldrake, 2002; Fuis et al., 2003; McGuire and Ben-Zion, 2005] Perturbations of a frictionless interface generate two generalized Rayleigh waves, which propagate in opposite directions with a phase velocity slightly less than the shear wave speed of either material. Associated with these waves are normal stress changes on the interface; the sign of the change is determined by the propagation direction of the wave. Adding an arbitrarily small amount of friction (as a 
constant $f$ ) couples shear and normal tractions on the interface, causing the wave that reduces normal stress to become unstable (and damping the other wave) [Ranjith and Rice, 2001].

[5] Andrews and Ben-Zion [1997] and Ben-Zion and Andrews [1998] demonstrated how this instability can generate slip pulses, which typically propagate unilaterally away from a localized nucleation region brought to instability by a transient decrease in effective normal stress. In their models, slip occurred at constant $f$, and the remote loading was such that the ratio of shear to effective normal stress on the fault was less than $f$ (except temporarily in the nucleation zone). Harris and Day [1997] also modeled slip between dissimilar elastic solids, but rather than keeping $f$ fixed, they allowed it to drop from static to dynamic values with increasing slip. Ruptures were nucleated by an initially forced bilateral expansion; after a short time, they propagated spontaneously. In this case, the ruptures took the form of bilaterally expanding cracks, rather than unilaterally propagating slip pulses. The bimaterial effect manifested itself by introducing asymmetry in both the rupture velocity of the two crack tips and the amplitude of slip velocities there.

[6] Ranjith and Rice [2001] performed a stability analysis of steady frictional sliding at constant $f$ between two dissimilar elastic half-spaces. The problem is linear and they considered perturbations in the form of spatial Fourier modes along the interface (i.e., sinusoidal variations in fields along the fault, with sections of the fault sliding slightly faster than the steady state slip velocity alternating with portions of the fault sliding at slower velocities). They found that when the material pair permits the existence of generalized Rayleigh waves in frictionless contact, modes of all wavelengths are unstable (i.e., the amplitude of the perturbation grows exponentially in time) when $f \neq 0$. The growth rate of the unstable Fourier modes is proportional to their wave number (which can be argued on dimensional grounds, since both the elastic response and friction law lack characteristic length or timescales). It follows that a general solution to the sliding problem, which can be written as a superposition of modes with different wave numbers, does not exist because the growth rate of the highest wave number modes becomes unbounded. Mathematically, the problem is said to be ill-posed. An important consequence of this is that numerical solutions to bimaterial problems in which at least some part of the sliding occurs at constant $f$ do not converge with mesh refinement. The set of ill-posed models include ruptures on a bimaterial interface with slip-weakening friction laws since such laws feature sliding at a constant dynamic friction coefficient after slip exceeds some critical distance.

[7] Practically speaking, this ill-posedness does not imply that frictional sliding between dissimilar elastic solids cannot occur in nature, but instead signifies that the mathematical model is pathological and that it must be modified (or, to be more precise, regularized) in such a way that the growth rate of Fourier modes is kept finite. (However, note that it is not necessary to stabilize any modes.) Ranjith and Rice [2001] showed that the bimaterial sliding problem with Coulomb friction could be regularized by requiring $\tau$ to approach $f \sigma$ over some finite slip or timescale; in particular, no instantaneous change of $\tau$ with $\sigma$ is permitted. This type of finite-slip evolution was observed in the high-speed sliding experiments of Prakash and Clifton [1993] and Prakash [1998]. However, other experiments [Linker and Dieterich, 1992; Richardson and Marone, 1999; Boettcher and Marone, 2004; Hong and Marone, 2005] at vastly slower slip speeds do show at least some degree of instantaneous coupling between $\tau$ and $\sigma$.

[8] Using a regularized Coulomb friction law, Cochard and Rice [2000] repeated the cases studied by Andrews and Ben-Zion [1997], finding convergent solutions that were similar in character to the original, unregularized ones. They also demonstrated that without regularization, numerical solutions that appeared sensible at a coarse resolution devolved into a sequence of short wavelength oscillations with mesh refinement (which adds to the solution shorter wavelength Fourier modes with larger growth rates). By employing a fixed resolution, one effectively prevents the introduction of any modes with wavelengths shorter than the grid spacing. For this reason, the numerical solutions by Andrews and Ben-Zion [1997] and others using the unregularized Coulomb friction law revealed many features that were later found to exist when the frictional response was regularized.

[9] It is important to note that the regularization procedure studied by Ranjith and Rice [2001] and Cochard and Rice [2000] does not stabilize any modes, but instead forces the growth rate to asymptotically vanish with decreasing wavelength. The numerical simulations by Ben-Zion and Huang [2002] suggest that solutions employing a regularized frictional response exhibit a finite-time divergence due to this instability; however, this divergence might be mitigated (or at least slowed) by permitting fault opening instead of allowing normal stresses to become tensile [Dalguer and Day, 2007]. Another process that apparently prevents divergence is plastic deformation of the medium, as demonstrated by Ben-Zion and Shi [2005]. Recent laboratory experiments by Xia et al. [2005] on bimaterial ruptures confirmed many of the phenomena described in the earlier numerical studies. In particular, the laboratory ruptures took the form of bilaterally expanding cracks whose tips traveled at different speeds, like those in the numerical simulations of Harris and Day [1997] and, as regards speed, the simulations of Cochard and Rice [2000]. It is not clear, however, if all properties seen in the experiments (e.g., bilateral expansion) would occur in natural earthquakes due to the artificial nucleation procedure employed in the experiments (and in numerical simulations). Other bimaterial rupture experiments with foam rubber blocks exhibit primarily unilateral propagation in the direction in which normal stress is expected to be reduced [Anooshehpoor and Brune, 1999].

[10] In this work, we study slip between dissimilar poroelastic solids, and show that for a wide range of material contrasts, steady frictional sliding (with a constant friction coefficient) is unstable to perturbations. This instability arises from alterations in pore pressure on the fault plane induced by the poroelastic response of fluid-saturated fault-bordering materials to spatially inhomogeneous slip on a permeable fault plane. This poroelastic effect (but not the related stability issues) was recognized by Rudnicki and Rice [2006], who studied it primarily in the context of slip between fluid-saturated materials that are elastically identical but have dissimilarity in poroelastic properties, espe- 

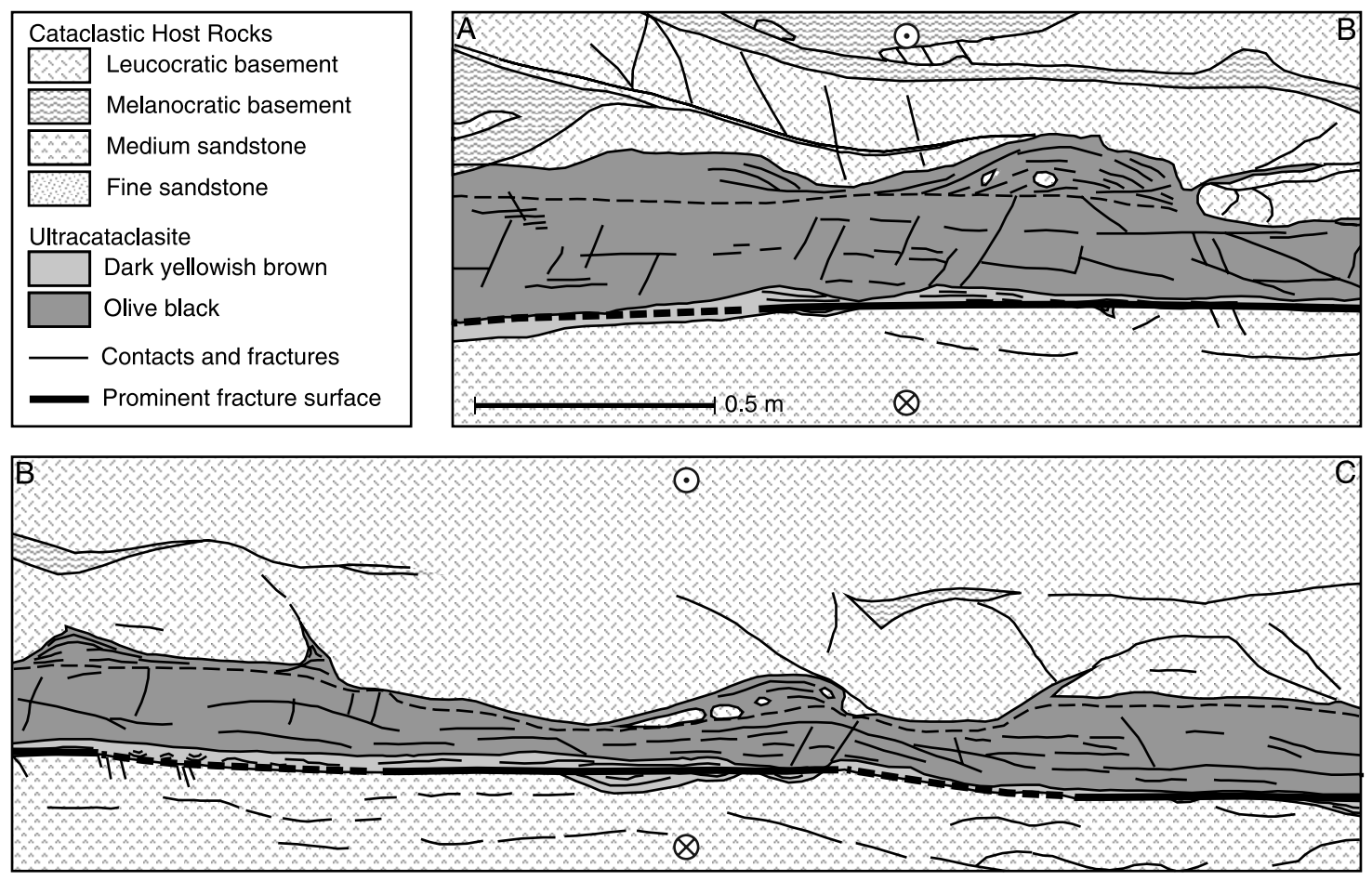

Figure 1. Structure of a slip-perpendicular exposure of the Punchbowl fault from Chester and Chester [1998]. Note that the prominent fracture surface in this case lies at the boundary between the ultracataclastic fault core and cataclastic damage zone.

cially permeability, within damage zones along the fault walls. Those authors also explored the mixture of near-fault poroelastic and far-field elastic dissimilarity in the context of steady state ruptures. Because Rudnicki and Rice [2006] focused solely on steady state solutions, it is impossible to assess issues related to the stability of those solutions and to frictional sliding problems in general. This prompted the current study.

[11] Geologic studies of faults [Chester and Chester, 1998; Heermance et al., 2003; Wibberley and Shimamoto, 2003; Chester et al., 2004] provide a basis for the model we develop (see Figure 1). Mature, well-slipped faults exhibit an ultracataclastic fault core within which lies an extremely localized slip surface. The fault core is several to tens of centimeters wide, and is surrounded by a broader damage zone of brecciated host rock (characterized by increased permeability relative to the fault core). The degree of damage decreases with increasing distance from the fault core, extending tens to hundreds of meters from the core. The damage zone is likely related to a region, hundreds of meters wide (at least near the surface), of low seismic velocities that has been inferred from seismic studies [ $L i$ et al., 1990; Ben-Zion et al., 2003].

[12] A large permeability contrast occurs across the transition between the fault core and the surrounding damage zone. In-situ and laboratory studies [Lockner et al., 2000; Wibberley, 2002; Wibberley and Shimamoto, 2003; Noda and Shimamoto, 2005] measure properties consistent with hydraulic diffusivities of $\sim \mathrm{mm}^{2} / \mathrm{s}$ within the fault core at confining pressures of $\sim 100 \mathrm{MPa}$ appropriate to the crustal seismogenic zone. These diffusivities are one or more orders of magnitude less than in the damage zone.
Rice [2006] presents a compilation of these measurements and notes that permeability within the fault core can be as much as three orders of magnitude less than that in the damage zone. The transition between the fault core and damage zone is abrupt, and the slip surface often juxtaposes these different materials [Chester and Chester, 1998; Chester et al., 2004; Dor et al., 2006].

[13] We next consider the poroelastic response of such a fault zone to stresses induced by slip during an earthquake. The response of fluid-saturated elastic media to applied stresses depends on the timescale of loading relative to the hydraulic diffusion time over spatial scales of interest. Compressional or extensional loads applied over times shorter than the diffusion time instantaneously increase or decrease the pore fluid pressure, respectively. This undrained response is followed by fluid flow driven by pressure differences. In the long time limit, the response is said to be drained. Fluid flow is negligible for seismic wave propagation since hydraulic diffusion lengths over a wave period are vastly smaller than the wavelength of the wave. Consequently, it is appropriate to interpret seismic inferences of elastic moduli as measurements of the undrained moduli.

[14] The traditionally considered elastic bimaterial problem of slip between solids with different elastic properties (density and elastic moduli) has neglected fluid effects. Poroelastic effects during in-plane rupture propagation between identical elastic materials were considered by Rudnicki and Koutsibelas [1991]. Compression of one side of the fault and extension of the other generates a pore pressure difference across the fault. Rudnicki and Koutsibelas [1991] treated the case of an impermeable fault, arguing that the 


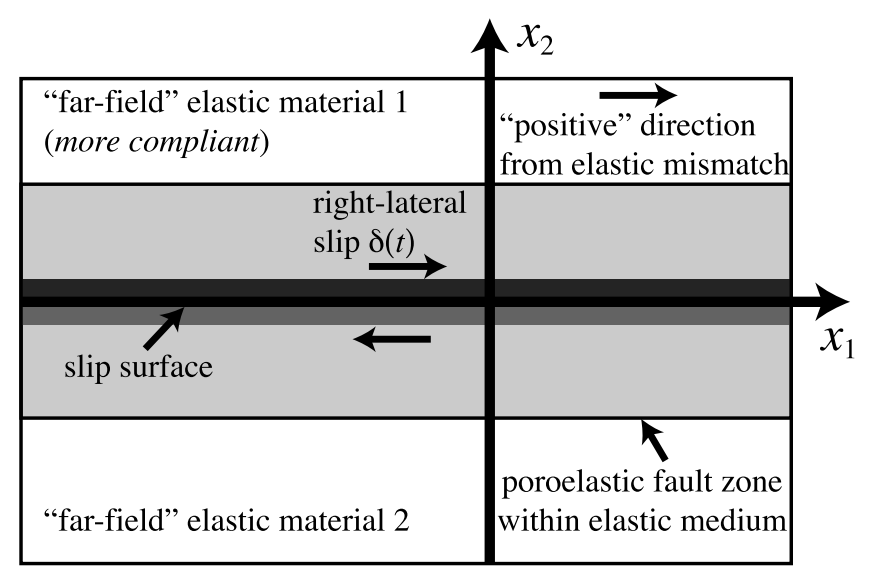

Figure 2. Poroelastic fault zone embedded within an elastic medium. The fault zone contains a planar slip surface bordered by damaged material. Material properties, both within the fault zone and in the far-field elastic medium, may be discontinuous across the fault. Heterogeneity in fault zone materials is permitted normal to the fault, provided that the width of the fault zone is smaller than all length scales characterizing the rupture (e.g., the extent of the cohesive zone or slip pulse). However, only the material properties sampled by hydraulic diffusion in the immediate vicinity of the slip surface are relevant to determining the poroelastic response.

pore pressure increase on the compressive side of the fault should be used in evaluating the effective stress used to determine the fault strength. The more general problem of a permeable fault has recently been investigated by Rudnicki and Rice [2006]. They recognized that fluid diffusion must be considered in the immediate vicinity of the slip plane in order to determine the pore pressure on the fault. The present work continues this effort by addressing issues related to the stability of frictional sliding and by exploring poroelastic bimaterial effects in the context of spontaneously propagating ruptures.

\section{Poroelastic Fault Zone Model}

[15] In our analysis we shall be concerned with how slip alters the stress, strain, and pore pressure fields within the fault zone and the surrounding material. We adopt the following notation: $\left[\sigma_{i j}\right]$ is the total stress tensor (positive in tension), $\left[\epsilon_{i j}\right]$ is the strain tensor, and $p$ is the pore pressure. As mentioned before, $\tau, \sigma$, and $\bar{\sigma}$ are, respectively, the shear stress, total normal stress, and effective normal stress on the fault (the latter two being positive in compression). Both stresses and pore pressure are to be understood as the sum of the initial, ambient fields existing prior to rupture (denoted with a subscript or superscript 0 , e.g., $\sigma_{i j}^{0}, \bar{\sigma}_{0}$ ) and the changes due to slip (denoted by the symbol $\Delta$, e.g., $\Delta \sigma_{i j}$, $\Delta p$ ). In contrast to this notation, $\epsilon_{i j}$ is the strain measured from the initially stressed state existing prior to rupture.

[16] Following Rudnicki and Rice [2006] we consider a poroelastic fault zone embedded in an elastic medium; within the fault zone at $x_{2}=0$ lies a planar slip surface (Figure 2). The material properties within the fault zone take on different values than those further away. It is well known that fault zones are highly heterogeneous in terms of material properties (e.g., elastic moduli and permeability); ultracataclastic fault core material is quite different than the bordering damaged material, and properties within this damage zone gradually approach those of the undamaged host rock with increasing distance from the fault. Our model permits this heterogeneity, but only under the assumption that the width of the fault zone (including the damage zone) is much smaller than all length scales characterizing the rupture (e.g., the extent of the cohesive zone or slip pulse). However, the relevant material properties within the fault zone are those confined to the narrow region surrounding the slip surface over which hydraulic diffusion is nonnegligible over the coseismic timescale. We refer to these bordering regions as damage fringes.

[17] Our model fits within a more general class of problems in which material properties vary with distance from the fault, which includes the studies of Harris and Day [1997] and Ben-Zion and Huang [2002] on how a lowvelocity zone of width comparable to the rupture scale influences rupture propagation. In our work, the material properties outside of the fault zone are taken to be spatially uniform, with values termed in the following as the far-field parameters (referring to material properties in the undamaged host rock surrounding the fault zone).

[18] In the case that the width of the fault zone (and damage fringes) is far less than all characteristic lengths of the rupture, certain components of the stress and strain fields evaluated within the fault zone are identical to what would have been calculated had the far-field elastic material extended to the slip surface (i.e., if the fault zone had not been present). Furthermore, the values of these stress and strain components are effectively constant over the scale of the fault zone, no matter the variation in material properties. In particular, continuity of traction across planes parallel to the slip surface implies that $\sigma_{i 2}(i=1,2,3)$ is effectively constant within the fault zone, and must furthermore be continuous across any transition in material properties that might occur at the boundary between the fault zone and farfield elastic medium.

[19] Our approach, then, is to match a far-field solution of the full elastodynamic equations (i.e., the solution to the problem in the absence of the fault zone) to an inner solution (valid only within the fault zone). The two solutions are matched by continuity of $\sigma_{22}$, as discussed above, and further by continuity of $\epsilon_{11}$ and $\epsilon_{33}$. The latter condition on the fault-parallel strains follows from continuity of the displacement field. (Note, however, that unlike $\sigma_{22}$, which is continuous across the fault, slip introduces a discontinuity of the fault-parallel strains across the slip surface.) Together with the constitutive relation, these conditions imply that certain other fields (such as $\sigma_{11}, \sigma_{33}$, and $\varepsilon_{22}$ ) are discontinuous, or may vary rapidly, when crossing into the fault zone and damage fringes. This is standard in the treatment of discontinuities in continuum mechanics [Hill, 1962].

[20] We first present our model in its full three-dimensional form, and then make the appropriate simplifications to specialize to the plane-strain case that comprises the remainder of this work. Furthermore, we treat all materials as isotropic. In the following, either superscripts + and - or, equivalently, subscripts 1 and 2 will be used to denote the $x_{2}>0$ and $x_{2}<0$ sides of the fault, respectively. The 
undrained pore pressure change, $\Delta p_{u}$, in response to stresses changes, $\Delta \sigma_{i j}$, on the two sides of the fault is

$$
\Delta p_{u}^{+}=-\frac{1}{3} B_{1} \Delta \sigma_{k k}^{+} \quad \text { and } \quad \Delta p_{u}^{-}=-\frac{1}{3} B_{2} \Delta \sigma_{k k}^{-},
$$

in which $B$ is Skempton's coefficient [Rice and Cleary, 1976; Wang, 2000], and summation is implied by repeated indices. Note that $\Delta p_{u}$ is essentially uniform in the faultnormal direction over the hydraulic diffusion length (i.e., the width of the damage fringes). It is convenient to reformulate equation (2) in terms of the fields that are continuous when entering the damage fringes from slightly more distant locations on the same side of the slip surface since these values can be obtained from solving the elasticity problem in the absence of the fault zone. This is accomplished using the stress-strain relation for undrained conditions [Rice and Cleary, 1976]:

$$
\begin{aligned}
& 2 \mu^{\mathrm{dmg}} \epsilon_{11}=\Delta \sigma_{11}-\frac{\nu_{u}}{1+\nu_{u}} \Delta \sigma_{k k} \\
& 2 \mu^{\mathrm{dmg}} \epsilon_{33}=\Delta \sigma_{33}-\frac{\nu_{u}}{1+\nu_{u}} \Delta \sigma_{k k},
\end{aligned}
$$

in which $v_{u}$ is the undrained Poisson's ratio within the damage fringes and the superscript "dmg" is used to distinguish the shear modulus within the damage fringes from its value far away from the fault. All poroelastic parameters (such as Skempton's coefficient) are to be understood as those measured within the damage fringes. Adding these equations and using $\Delta \sigma_{11}+\Delta \sigma_{33}=\Delta \sigma_{k k}-$ $\Delta \sigma_{22}$, we arrive at an expression for $\Delta \sigma_{k k}$ in terms of $\epsilon_{11}$, $\epsilon_{33}$, and $\Delta \sigma_{22}$. Using this in equation (2), we obtain

$$
\begin{aligned}
& \Delta p_{u}^{+}=-\frac{Q_{1}}{2}\left[2 \mu_{1}^{\mathrm{dmg}}\left(\epsilon_{11}^{+}+\epsilon_{33}^{+}\right)+\Delta \sigma_{22}\right] \\
& \Delta p_{u}^{-}=-\frac{Q_{2}}{2}\left[2 \mu_{2}^{\mathrm{dmg}}\left(\epsilon_{11}^{-}+\epsilon_{33}^{-}\right)+\Delta \sigma_{22}\right],
\end{aligned}
$$

in which we have introduced the simplifying notation

$$
Q_{i}=\frac{2\left(1+\nu_{u i}\right)}{3\left(1-\nu_{u i}\right)} B_{i} \quad(i=1,2) .
$$

The advantage of equation (4) over equation (2) is that values of the fields appearing in equation (4) may be taken directly from the far-field solution (i.e., the values of the fields that would exist if the medium was uniform up to the slip surface).

[21] The pressure difference across the fault induces flow according to Darcy's law:

$$
q=-\frac{\rho_{f} k}{\eta_{f}} \frac{\partial p}{\partial x_{2}}
$$

where $q$ is the fluid flux, $\rho_{f}$ is the fluid mass density, $k$ is the permeability, and $\eta_{f}$ is the fluid viscosity. Together with equation (6), fluid mass conservation implies that, at each point on the fault, the difference in pore pressure from its undrained value satisfies a homogeneous diffusion equation:

$$
\left(\frac{\partial}{\partial t}-\alpha_{h y} \frac{\partial^{2}}{\partial x_{2}^{2}}\right)\left[p\left(x_{2}, t\right)-p_{u}(t)\right]=0 .
$$

Here $\alpha_{h y}=k / \eta_{f} \beta$ is the hydraulic diffusivity and $\beta$ is a storage coefficient; these parameters are discussed further

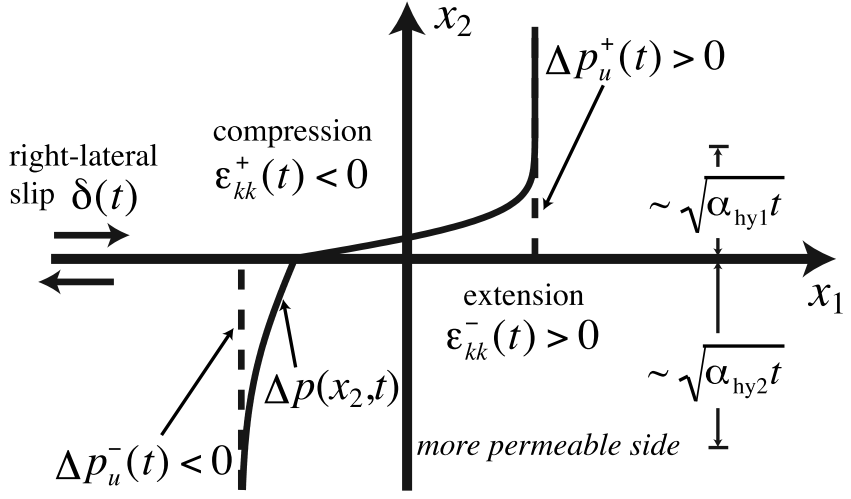

Figure 3. Poroelastic response within the damage fringes to the application of far-field stresses. In-plane slip causes one side of the fault to be compressed and the other to be extended. The immediate, undrained change in pore pressure, $\Delta p_{u}$, is consequently of opposite sign on either side of the fault. The pressure difference induces fluid diffusion, subject to continuity of pore pressure, $p$, and fluid flux across the fault. Any contrast in material properties across the fault results in a nonzero alteration of pore pressure on the fault plane.

when we consider representative parameter values. Note that diffusion parallel to the fault is negligible during the rupture process. Consider a rupture characterized by a minimum length scale $\lambda$ over which along-fault gradients in the stress fields induce gradients in pore pressure. Fluid flow occurs only over the time that this spatial gradient persists over some section of the fault. For a rupture moving at speed $v_{r}$, this time is $\lambda v_{r}$. The diffusion length over this timescale is $\sqrt{\alpha_{h y} \lambda / v_{r}}$. If this diffusion length is much smaller than $\lambda$, then along-fault fluid flow is negligible. Using $v_{r} \sim \mathrm{km} / \mathrm{s}$ and $\alpha_{h y} \sim \mathrm{mm}^{2} / \mathrm{s}$, we estimate that only if $\lambda$ is less than $\sim \mathrm{nm}$ is along-fault fluid flow significant. It follows that variations in fields parallel to the fault influence only the undrained poroelastic response.

[22] Rudnicki and Rice [2006] have shown that the solution to this one-dimensional diffusion problem, subject to continuity of pore pressure and fluid flux across the fault, yields the resulting change in pore pressure on the fault:

$$
\Delta p=\zeta_{1} \Delta p_{u}^{+}+\zeta_{2} \Delta p_{u}^{-},
$$

where $\zeta_{i}=Z_{i} /\left(Z_{1}+Z_{2}\right)$ and $Z_{i}=\sqrt{k_{i} \beta_{i}}$ for $i=1,2$. Note that $0 \leq \zeta_{i} \leq 1$, with the limits of this range corresponding to one side being far more permeable than the other. When $k_{1} \ll k_{2}$, then $\zeta_{1}=0$ and $\zeta_{2}=1$; conversely, if $k_{1} \gg k_{2}$, then $\zeta_{1}=1$ and $\zeta_{2}=0$. A schematic of the poroelastic response is shown in Figure 3.

[23] Combining equations (4) and (8) yields

$$
\Delta p=-q_{0} \Delta \sigma_{22}-q_{1} \mu_{1}\left(\epsilon_{11}^{+}+\epsilon_{33}^{+}\right)-q_{2} \mu_{2}\left(\epsilon_{11}^{-}+\epsilon_{33}^{-}\right),
$$

where

$$
\begin{aligned}
& q_{0}=\frac{1}{2}\left(\zeta_{1} Q_{1}+\zeta_{2} Q_{2}\right) \\
& q_{i}=\zeta_{i} m_{i} Q_{i} \quad(i=1,2)
\end{aligned}
$$


Table 1. Representative Poroelastic Parameters for a Fault Dividing More and Less Damaged Materials Consistent With a Factor-of-Ten Contrast in Permeability ${ }^{\mathrm{a}}$

\begin{tabular}{|c|c|c|}
\hline Parameter & More Damaged Side & Less Damaged Side \\
\hline $\begin{array}{l}\text { Permeability } \\
\left(k_{1} \text { or } k_{2}\right)\end{array}$ & $k$ & $k / 10$ \\
\hline $\begin{array}{l}\text { Storage factor } \\
\left(\beta_{1} \text { or } \beta_{2}\right)\end{array}$ & $\beta$ & $\beta / 2$ \\
\hline $\begin{array}{l}\text { Skempton's coefficient } \\
\left(B_{1} \text { or } B_{2}\right)\end{array}$ & 0.6 & 0.4 \\
\hline $\begin{array}{l}\text { Poisson's ratio } \\
\left(v_{u 1} \text { or } v_{u 2}\right)\end{array}$ & 0.3 & 0.3 \\
\hline $\begin{array}{l}\text { Shear modulus ratio } \\
\left(\mu_{1} \mathrm{dmg} / \mu_{1} \text { or } \mu_{2}{ }^{\mathrm{dmg}} / \mu_{2}\right)\end{array}$ & 0.25 & 0.5 \\
\hline $\begin{array}{l}q_{1} \text { or } q_{2} \\
q_{0}\end{array}$ & \multicolumn{2}{|c|}{0.35} \\
\hline
\end{tabular}

${ }^{\mathrm{a}} k$ and $\beta$ are the permeability and storage factor of the more damaged side of the fault; these values are left unspecified since only the contrast in these properties across the fault is important. The three nondimensional parameters determining the poroelastic bimaterial response $\left(q_{0}, q_{1}\right.$, and $\left.q_{2}\right)$ are defined in equations (9) and (10).

and

$$
m_{i}=\frac{\mu_{i}^{\mathrm{dmg}}}{\mu_{i}} \quad(i=1,2) .
$$

Note that, in equations (9) and (11), $\mu_{1}$ and $\mu_{2}$ are the farfield shear moduli far outside the damage fringes.

[24] The various terms in equation (9) merit further discussion. The first, proportional to $\Delta \sigma_{22}$, is the poroelastic correction to the normal stress change resulting from an elastic mismatch. Because $0 \leq q_{0}<1$ (which follows from the restriction that $0 \leq B<1$ ), this correction always decreases, by the factor $1-q_{0}$, the magnitude of the effective normal stress change that would be predicted if poroelastic effects were ignored. The physical significance of this term is straightforward. If a uniform compressive normal stress is applied to a poroelastic fault zone, compression of the pores increases the pressure of the fluid within them. Conversely, extension of the fault zone material generates a suction and decreases pore pressure. The result is that any normal stress applied to the fault zone is counteracted by a change in pore pressure, with a magnitude equal to $q_{0}$ times the applied normal stress change.

[25] The second and third terms in equation (9) depend on the undrained pore pressure changes caused by fault-parallel strains and subsequent fluid diffusion across the fault. There are two cases to consider here, which we discuss in the twodimensional plane-strain limit $\left(\epsilon_{33}=0\right)$. First, if the fault is not slipping but there is a gradient in the fault-parallel displacement field (as occurs prior to the arrival of the rupture front if there is an elastic mismatch across the fault), then $\epsilon_{11}^{+}=\epsilon_{11}^{-}$and the change in pore pressure from these strains is of opposite sign to the strains themselves. The reason for this is identical to that explaining the normal stress changes. When the fault is slipping, then $\epsilon_{11}^{+}$and $\epsilon_{11}^{-}$ have opposite signs, and the pore pressure response is similar to that illustrated in Figure 3. Like the first term in equation (9), the terms associated with fault-parallel strains persist even if there is no poroelastic mismatch across the fault, provided that a far-field elastic dissimilarity results in unequal magnitudes of the fault-parallel strains.
[26] What are representative values of $q_{0}, q_{1}$, and $q_{2}$ ? The answer depends on the precise location of the slip surface within the fault zone. Geologic studies suggest that it is not uncommon for slip to localize at the interface between the relatively impermeable fault core material and more permeable damaged rock [Chester and Chester, 1998; Chester et al., 2004; Dor et al., 2006] An example of this is shown in Figure 1. In addition, numerical simulations that permit slip on any of a set of parallel surfaces having identical friction coefficients indicate a tendency for slip to select a material interface, when that interface is the one which most reduces $\bar{\sigma}$ [Brietzke and Ben-Zion, 2006]. It thus seems reasonable to suppose a factor-of-ten permeability contrast across the slip surface; in fact, permeabilities inside and outside the fault core may be several orders of magnitude different [Lockner et al., 2000; Wibberley, 2002; Wibberley and Shimamoto, 2003; Noda and Shimamoto, 2005]. Accompanying the permeability contrast are contrasts in other material properties, arising from a different degree of damage across the fault or simply occurring when the fault runs between two types of rock. Similar estimates of poroelastic properties within a fault zone are given by Cocco and Rice [2002]. On both sides of the fault, we take an undrained Poisson's ratio of 0.3. It is well known that damage, in the form of distributed microcracks, leads to a reduction in elastic moduli [O'Connell and Budiansky, 1974; Budiansky and O'Connell, 1976]. Seismic estimates of wave speed in the low-velocity zone of faults indicate about a $50 \%$ reduction in moduli, assuming no reduction in density [Li et al., 1990; Ben-Zion et al., 2003]. Those studies sample the fault structure over much longer scales than those of the damage fringes considered here, and it seems quite probable that elastic moduli might even be further reduced within the portion of the damage zone immediately adjacent to the fault core. Consequently, we assume that the shear modulus is reduced to $50 \%$ and $25 \%$ of its far-field value on the less and more damaged sides of the fault, respectively.

[27] The storage factor, $\beta$, is more precisely written in terms of the compressibility of the pore fluid, $\beta_{f}$, the pore space expansivity, $\beta_{n}$, and the porosity, $n$, as $\beta=n\left(\beta_{f}+\beta_{n}\right)$ [Rice, 2006]. Rice presents a compilation of measurements and deduces representative values of $\beta$ for both damaged and undamaged fault material; see his Tables 1 and 2. Just as we have taken a factor-of-two contrast in shear moduli across the fault, we also take a factor-of-two contrast in $\beta$.

[28] The final parameter that enters our model is Skempton's coefficient, $B$. Using a compilation of measurements

Table 2. Model Parameters, Similar to Those Used in the Southern California Earthquake Center Validation Tests [Harris and Archuleta, 2004], but With the Initial Shear Stress Reduced to Prevent Supershear Rupture Speeds

\begin{tabular}{llc}
\hline S-wave speed & $c_{\mathrm{s} 2}$ & $3.464 \mathrm{~km} / \mathrm{s}$ \\
P-wave speed & $c_{\mathrm{p} 2}$ & $6 \mathrm{~km} / \mathrm{s}$ \\
Shear modulus & $\mu_{2}$ & $32.04 \mathrm{GPa}$ \\
Static friction coefficient & $F_{\mathrm{s}}$ & 0.677 \\
Dynamic friction coefficient & $F_{\mathrm{d}}$ & 0.525 \\
Slip-weakening distance & $D_{\mathrm{c}}$ & $0.4 \mathrm{~m}$ \\
Regularization timescale & $t^{*}$ & $0.01 \mathrm{~s}$ \\
Initial shear stress & $\sigma_{21}^{0}$ & $67.56 \mathrm{MPa}$ \\
Initial effective normal stress & $\bar{\sigma}_{0}$ & $120 \mathrm{MPa}$ \\
\hline
\end{tabular}


from the literature, Rice and Cleary [1976] estimate values of $B$ for rocks between 0.5 and 0.9 . Recent laboratory experiments have measured poroelastic properties of sandstone and limestone and their dependence on stress conditions [Hart and Wang, 1995; Lockner and Stanchits, 2002; Lockner and Beeler, 2003]. Here we are concerned primarily with the fault core materials in the immediate vicinity of the slip surface. E. Templeton (personal communication, 2007) evaluated $B$ from data for ultracataclastic fault gouge compiled by Rice [2006] and found typical values around $0.6-0.8$ at temperatures and pressures representative of those at midseismogenic depth. We adopt slightly more conservative values and use $B=0.6$ and 0.4 for the more and less damaged sides, respectively. A compilation of our parameter values is given in Table 1.

[29] We should also point out that, in selecting the value of pore pressure to be used in the effective stress law equation (1) to determine fault strength, we have chosen the value of $p$ at $x_{2}=0$. This is consistent with our assumption that slip occurs on a mathematical plane, and it should furthermore be valid for finite-width shear zones provided that the width of the shear zone is far smaller than the hydraulic diffusion length over the timescales of interest. It remains an open question as to how the effective stress law should be written (i.e., what value of $p$ should be used when evaluating fault strength) if this condition is not met.

\section{Stability of Frictional Sliding}

[30] It is known that frictional sliding between dissimilar elastic materials is unstable for a broad range of elastic contrasts and friction coefficients [Ranjith and Rice, 2001], and the similar nature of the poroelastic bimaterial response studied by Rudnicki and Rice [2006] suggests that frictional sliding between dissimilar poroelastic materials might also be unstable. However, the steady state analysis of Rudnicki and Rice [2006] cannot be used to address whether or not this is true. Additionally, the stability analysis of Ranjith and Rice [2001] provided much insight into the behavior of numerical simulations of elastic bimaterial ruptures. Consequently, an appropriate starting point for quantitatively analyzing poroelastic bimaterial effects and their interaction with far-field elastic bimaterial effects is a stability analysis of steady frictional sliding. This analysis further permits us to identify interesting parameter ranges to be investigated in later numerical studies of spontaneously propagating ruptures.

[31] We address stability of steady frictional sliding by considering the response to Fourier mode perturbations with wave number $k$ (not to be confused with the permeabilities of the two sides of the fault, $k_{1}$ and $k_{2}$ ) about a constant slip velocity $V$ :

$$
\begin{aligned}
\sigma_{21}\left(x_{1}, t\right) & =\sigma_{21}^{0}+T_{1}(t) e^{i k x_{1}} \\
\sigma_{22}\left(x_{1}, t\right) & =\sigma_{22}^{0}+T_{2}(t) e^{i k x_{1}} \\
u_{1}^{ \pm}\left(x_{1}, t\right) & = \pm V t / 2+U_{1}^{ \pm}(t) e^{i k x_{1}} \\
u_{2}^{ \pm}\left(x_{1}, t\right) & =U_{2}^{ \pm}(t) e^{i k x_{1}} \\
p\left(x_{1}, t\right) & =p_{0}+P(t) e^{i k x_{1}},
\end{aligned}
$$

where $u_{i}$ is the displacement field. The unperturbed state corresponds to one of uniform stress in the medium and constant pore pressure on the fault, subject to the constraint that the fault slides at a constant friction coefficient $f$; i.e., $\sigma_{21}^{0}=f\left(-\sigma_{22}^{0}-p_{0}\right)$.

\subsection{Slip Response to Shear Traction Perturbations}

[32] After Laplace transforming time, using the notation

$$
\hat{g}(s)=\int_{0}^{\infty} g(t) e^{-s t} d t
$$

the following relationship exists between displacement and stress perturbations on the fault [Geubelle and Rice, 1995; Ranjith and Rice, 2001]:

$$
\left[\begin{array}{c}
\hat{U}_{1}^{ \pm} \\
\hat{U}_{2}^{ \pm}
\end{array}\right]=\left[\begin{array}{ll} 
\pm \hat{C}_{11}^{ \pm} & \hat{C}_{12}^{ \pm} \\
\hat{C}_{21}^{ \pm} & \pm \hat{C}_{22}^{ \pm}
\end{array}\right]\left[\begin{array}{l}
\hat{T}_{1} \\
\hat{T}_{2}
\end{array}\right]
$$

in which

$$
\begin{aligned}
& \hat{C}_{11}=-\frac{1}{\mu|k|} \frac{\alpha_{s}\left(1-\alpha_{s}^{2}\right)}{R} \\
& \hat{C}_{22}=-\frac{1}{\mu|k|} \frac{\alpha_{p}\left(1-\alpha_{s}^{2}\right)}{R} \\
& \hat{C}_{12}=-\frac{1}{i \mu k} \frac{2 \alpha_{s} \alpha_{p}-\left(1+\alpha_{s}^{2}\right)}{R}=-\hat{C}_{21} .
\end{aligned}
$$

The superscripts + and - in the above expressions for $\hat{C}_{i j}$ indicate that they are to be evaluated using far-field material properties relevant to the positive or negative side of the fault, respectively. A similar notation applies in later expressions as well. We also define

$$
\begin{aligned}
& \alpha_{p}=\sqrt{1-s^{2} / k^{2} c_{p}^{2}} \\
& \alpha_{s}=\sqrt{1-s^{2} / k^{2} c_{s}^{2}},
\end{aligned}
$$

in which $c_{\mathrm{p}}$ and $c_{\mathrm{s}}$ are the $\mathrm{P}$ - and $\mathrm{S}$-wave speeds, respectively.

[33] Slip and opening are defined as $\delta_{1}\left(x_{1}, t\right)=u_{1}^{+}\left(x_{1}, t\right)-$ $u_{1}^{-}\left(x_{1}, t\right)$ and $\delta_{2}\left(x_{1}, t\right)=u_{2}^{+}\left(x_{1}, t\right)-u_{2}^{-}\left(x_{1}, t\right)$, respectively. With the notation $\delta_{1}\left(x_{1}, t\right)=D_{1}(t) \exp ^{i k x_{1}}$ and $\delta_{2}\left(x_{1}, t\right)=$ $D_{2}(t) \exp ^{i k x_{1}}$, expressions from both sides of the fault may be combined as

$$
\left[\begin{array}{l}
\hat{D}_{1} \\
\hat{D}_{2}
\end{array}\right]=\left[\begin{array}{ll}
\hat{K}_{11} & \hat{K}_{12} \\
\hat{K}_{21} & \hat{K}_{22}
\end{array}\right]\left[\begin{array}{l}
\hat{T}_{1} \\
\hat{T}_{2}
\end{array}\right]
$$

with

$$
\begin{aligned}
& \hat{K}_{11}=\hat{C}_{11}^{+}+\hat{C}_{11}^{-} \\
& \hat{K}_{22}=\hat{C}_{22}^{+}+\hat{C}_{22}^{-} \\
& \hat{K}_{12}=\hat{C}_{12}^{+}-\hat{C}_{12}^{-}=-\hat{K}_{21} .
\end{aligned}
$$

The inverse of this expression is

$$
\left[\begin{array}{l}
\hat{T}_{1} \\
\hat{T}_{2}
\end{array}\right]=\left[\begin{array}{ll}
\hat{M}_{11} & \hat{M}_{12} \\
\hat{M}_{21} & \hat{M}_{22}
\end{array}\right]\left[\begin{array}{l}
\hat{D}_{1} \\
\hat{D}_{2}
\end{array}\right]
$$

in which

$$
\begin{aligned}
& \hat{M}_{11}=\hat{K}_{22} / D \\
& \hat{M}_{22}=\hat{K}_{11} / D \\
& \hat{M}_{12}=-\hat{K}_{12} / D=-\hat{M}_{21}
\end{aligned}
$$


and

$$
D=\hat{K}_{22} \hat{K}_{11}-\hat{K}_{12} \hat{K}_{21}
$$

It will furthermore be necessary to have an expression for the fault-parallel strain $\epsilon_{11}^{ \pm}\left(x_{1}, t\right)=E_{11}^{ \pm}(t) \exp ^{i k x_{1}}$ :

$$
\hat{E}_{11}^{ \pm}=\left[\begin{array}{ll}
i k & 0
\end{array}\right]\left[\begin{array}{c}
\hat{U}_{1}^{ \pm} \\
\hat{U}_{2}^{ \pm}
\end{array}\right]
$$

Combining equation (22) with equation (14) yields

$$
\mu^{ \pm} \hat{E}_{11}^{ \pm}=\left[\begin{array}{ll} 
\pm \hat{J}_{1}^{ \pm} & \hat{J}_{2}^{ \pm}
\end{array}\right]\left[\begin{array}{l}
\hat{T}_{1} \\
\hat{T}_{2}
\end{array}\right]
$$

where

$$
\begin{aligned}
& \hat{J}_{1}=i k \mu \hat{C}_{11}=-\frac{i k}{|k|} \frac{\alpha_{s}\left(1-\alpha_{s}^{2}\right)}{R} \\
& \hat{J}_{2}=i k \mu \hat{C}_{12}=\frac{1+\alpha_{s}^{2}-2 \alpha_{s} \alpha_{p}}{R} .
\end{aligned}
$$

[34] At this point, we can write an expression for the change in pore pressure as set by the undrained response and its mitigation by diffusion within the damage fringes. This is accomplished by substituting equation (23) into the transform of equation (9) and using the plane strain condition $\epsilon_{33}^{ \pm}=0$. The result is

$$
\hat{P}=-\left[\begin{array}{ll}
\hat{L}_{1} & \hat{L}_{2}+q_{0}
\end{array}\right]\left[\begin{array}{l}
\hat{T}_{1} \\
\hat{T}_{2}
\end{array}\right],
$$

with

$$
\begin{aligned}
& \hat{L}_{1}=q_{1} \hat{J}_{1}^{+}-q_{2} \hat{J}_{1}^{-} \\
& \hat{L}_{2}=q_{1} \hat{J}_{2}^{+}+q_{2} \hat{J}_{2}^{-} .
\end{aligned}
$$

[35] Now consider adding a perturbation, $Q(t) \exp ^{i k x_{1}}$, to the shear stress $\sigma_{21}\left(x_{1}, t\right)$. This is achieved by replacing $\hat{T}_{1}$ in equations (19) and (25) with $\hat{T}_{1}-\hat{Q}$ :

$$
\begin{aligned}
{\left[\begin{array}{l}
\hat{T}_{1} \\
\hat{T}_{2}
\end{array}\right] } & =\left[\begin{array}{ll}
\hat{M}_{11} & \hat{M}_{12} \\
\hat{M}_{21} & \hat{M}_{22}
\end{array}\right]\left[\begin{array}{l}
\hat{D}_{1} \\
\hat{D}_{2}
\end{array}\right]+\left[\begin{array}{c}
\hat{Q} \\
0
\end{array}\right] \text { and } \\
\hat{P} & =-\left[\begin{array}{ll}
\hat{L}_{1} & \hat{L}_{2}+q_{0}
\end{array}\right]\left[\begin{array}{c}
\hat{T}_{1}-\hat{Q} \\
\hat{T}_{2}
\end{array}\right] .
\end{aligned}
$$

These two equations, equations (27) and (28), complete the description of the elastic and poroelastic response. In order to solve for the slip response to the perturbation, we must apply frictional boundary conditions.

\subsection{Sliding With Coulomb Friction}

[36] The relevant boundary conditions for sliding under a constant coefficient of friction $f$ and no opening are

$$
\begin{gathered}
\hat{T}_{1}=-f\left(\hat{T}_{2}+\hat{P}\right) \\
\hat{D}_{2}=0 .
\end{gathered}
$$

Using equations (27) and (28), and solving for slip, we obtain

$$
\begin{aligned}
\hat{D}_{1} & =-\frac{\hat{Q}}{\left(1-f \hat{L}_{1}\right) \hat{M}_{11}+f\left(1-\hat{L}_{2}-q_{0}\right) \hat{M}_{21}} \\
& =-\frac{\hat{Q} D}{\left(1-f \hat{L}_{1}\right) \hat{K}_{22}+f\left(1-\hat{L}_{2}-q_{0}\right) \hat{K}_{12}} \\
& =-\frac{\hat{Q} D}{\hat{K}_{22}+f\left[\left(1-\hat{L}_{2}-q_{0}\right) \hat{K}_{12}-\hat{L}_{1} \hat{K}_{22}\right]}
\end{aligned}
$$

Note that when $f=0$, the denominator is just $\hat{K}_{22}$, and $\hat{K}_{22}=$ 0 has as its solution the generalized Rayleigh wave [Ranjith and Rice, 2001]. When $B_{1}=B_{2}=0$, the poroelastic coupling vanishes. In this limit, $\hat{L}_{1}=\hat{L}_{2}=q_{0}=0$ and the denominator becomes $\hat{K}_{22}+f \hat{K}_{12}$, as determines the purely elastic bimaterial response.

\subsection{Instability When Generalized Rayleigh Wave Exists}

[37] In the following discussion, we view $\hat{K}_{i j}$ and related quantities as functions of $S=s / k$. (This is a slightly different notation than that used by Ranjith and Rice [2001] who wrote the perturbation as $\exp (i k x-p t)$ and defined $s=p / k$. Our $s$ corresponds to their $p$, and our $S$ is their $s$.)

[38] When $f=0, \hat{K}_{22}\left(S_{0}\right)=0$ for $S_{0}= \pm i c_{G R}$. This root corresponds to the generalized Rayleigh wave, and $c_{G R}$ is the generalized Rayleigh speed. For small $f$, we expand the root as $S=S_{0}+f S_{1}+O\left(f^{2}\right)$, and identify

$$
S_{1}=-\left(1-\hat{L}_{2}-q_{0}\right) \hat{K}_{12} /\left.\hat{K}_{22}^{\prime}\right|_{S=S_{0}} .
$$

Ranjith and Rice [2001] have shown that both $\hat{K}_{12}\left(S_{0}\right)$ and $\hat{K}_{22}^{\prime}\left(S_{0}\right)$ are purely imaginary at $S_{0}$; therefore $S_{1}=$ $\left(1-\hat{L}_{2}-q_{0}\right) \times$ (purely real). Note that

$$
\hat{L}_{2}=q_{1} \frac{1+\alpha_{s 1}^{2}-2 \alpha_{s 1}-\alpha_{p 1}}{R_{1}}+q_{2} \frac{1+\alpha_{s 2}^{2}-2 \alpha_{s 2} \alpha_{p 2}}{R_{2}}
$$

is purely real at $S_{0}$, as is $q_{0}$. Ranjith and Rice [2001] have also shown that

$$
\hat{K}_{12}\left( \pm i c_{G R}\right) / \hat{K}_{22}^{\prime}\left( \pm i c_{G R}\right)= \pm \hat{K}_{12}\left(i c_{G R}\right) / \hat{K}_{22}^{\prime}\left(i c_{G R}\right)
$$

so that $S_{1}$ is purely real and of opposite sign for $S_{0}= \pm i c_{G R}$. Hence as $f$ increases from zero, the two poles move in opposite directions parallel to the real $S$-axis, one into the stable half plane and the other into the unstable half plane. The poroelastic effect alters the elastic bimaterial stability properties, at least in the context of this linearized expansion, by either increasing or decreasing the growth rate by the factor $1-\hat{L}_{2}-q_{0}$.

[39] To further explore the interactions between the poroelastic and elastic mismatch effect requires a numerical solution for the poles of equation (31). We consider two cases which result from choosing the compliant side of the fault to be either more or less permeable than the stiff side. The two effects oppose each other if the stiff side is more permeable, and enhance each other if the compliant side is more permeable.

[40] When the effects oppose each other, the question arises as to which determines the propagation direction of 
Opposing Effects

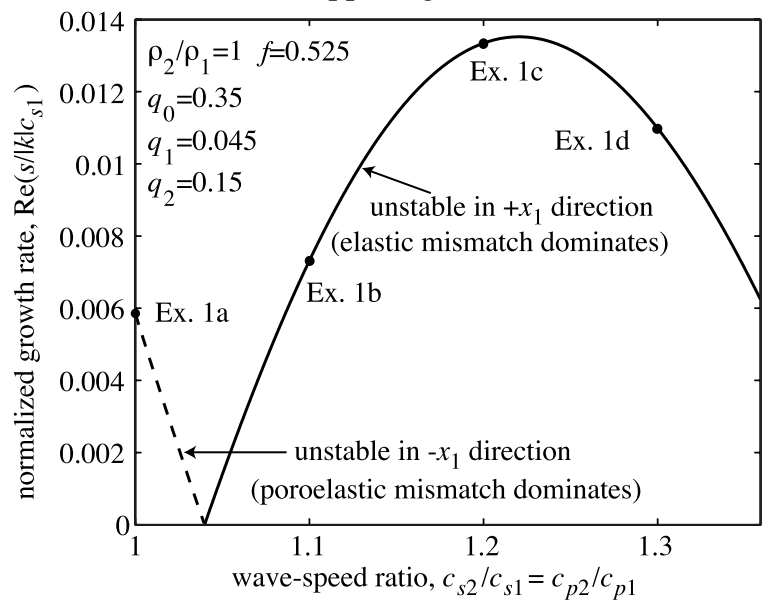

Enhancing Effects

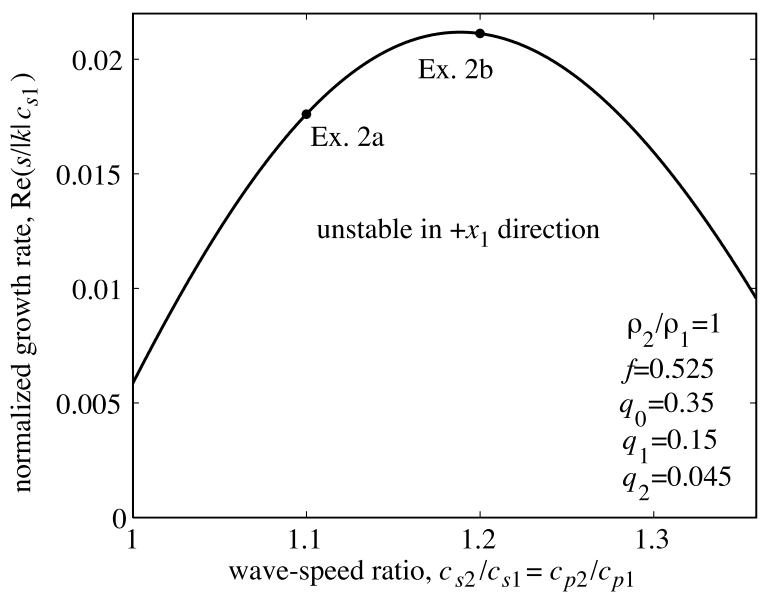

Figure 4. Growth rate of the unstable mode for (left) opposing poroelastic and elastic effects with the stiff side more permeable and (right) enhancing poroelastic and elastic effects with the compliant side more permeable.

the unstable waves. We consider here the case of a fixed poroelastic mismatch (consistent with a factor of ten contrast in permeability; see Table 1) and vary the elastic mismatch. The left panel of Figure 4 shows the growth rate of the unstable mode as a function of wave speed ratio for the case of no density contrast. With no wave speed contrast, the poroelastic effect determines the propagation direction of the unstable mode. As the wave speed contrast increases, the elastic mismatch effect begins to oppose the poroelastic effect, and at about a $5 \%$ contrast in wave speeds, the two effects precisely balance each other. Further increasing the elastic mismatch reverses the propagation direction of the unstable mode. A plot of the growth rate for enhancing effects is shown in the right panel of Figure 4.

[41] To summarize, when the generalized Rayleigh wave exists in frictionless contact, sliding with an arbitrarily small coefficient of friction is unstable, no matter the mismatch in poroelastic properties. However, the growth rate of the instability vanishes as $f \rightarrow 0$ and also in the limit of identical material properties.

\subsubsection{Regions of Instability}

[42] We numerically solved for the critical coefficient of friction at which sliding destabilizes as a function of farfield density ratio, far-field wave speed ratio, and degree of poroelastic mismatch. Poisson's ratio in the far-field elastic material is $1 / 4$ in all cases. The poroelastic mismatch is specified in terms of the parameters $q_{1}$ and $q_{2}$ that are defined in equation (10). The result for a density ratio of 1.2 is shown in Figure 5.

[43] As in the case of a purely elastic mismatch [Ranjith and Rice, 2001], there exists a region of stability centered around a wave speed contrast of about $50-100 \%$ for friction coefficients less than about unity. Outside of this region, steady sliding is unstable, with the instability taking the form of exponentially growing propagating wave modes. The edges of the stable region are delimited by instability associated with different unstable modes (having different phase velocities). This was investigated in detail by Ranjith and Rice [2001], and we find that the same pattern exists when poroelastic bimaterial effects are included. The pri- mary effect of the poroelastic response, even in the absence of any poroelastic contrast, is to increase the region of stability (as is evident in the difference between the solid and dashed black curves in Figure 5). This is simply a manifestation of the difference between using normal stress and effective normal stress when evaluating fault strength. In the absence of any poroelastic contrast, the effective

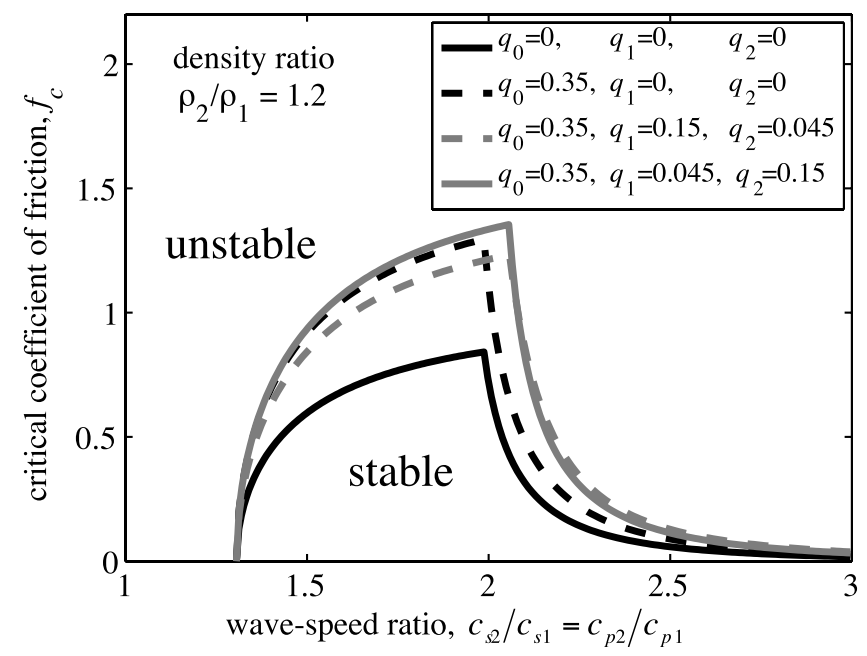

Figure 5. Regions of instability for a density ratio of 1.2. The solid black line corresponds to the case of no poroelastic response and the dashed black line is for the case with the response included, assuming that poroelastic properties are homogeneous. Adding the poroelastic effect stabilizes sliding over a larger range of friction coefficients. Cases for which one side is more permeable than the other are shown by the solid gray and dashed gray lines. When the stiff side is more permeable, the poroelastic effect opposes the elastic bimaterial effect and the region of stability expands (solid gray line). When the compliant side is more permeable, the poroelastic and elastic bimaterial effects act in concert and the region of stability diminishes (dashed gray line). 


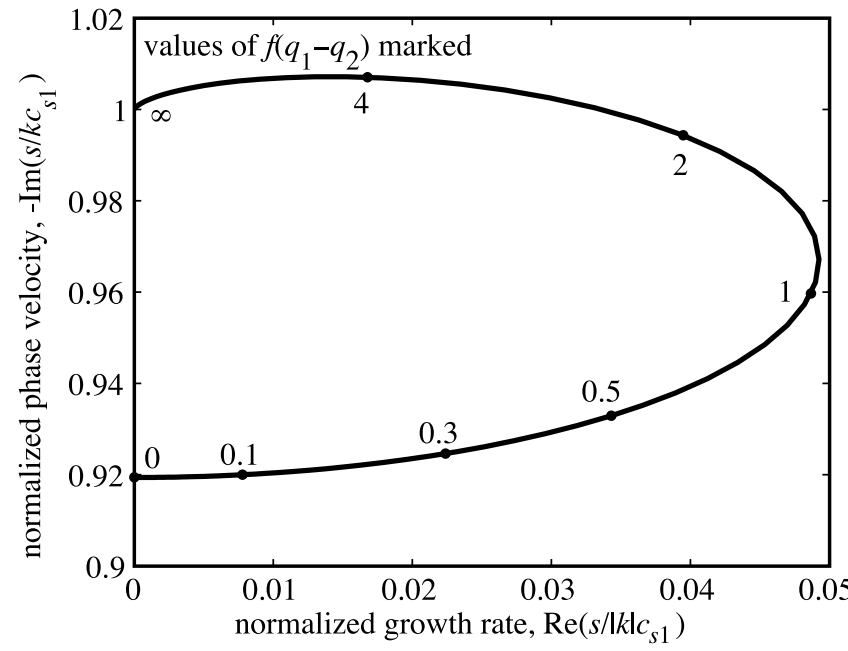

Figure 6. Growth rate and phase velocity of the perturbation for identical elastic properties, shown as a function of $f\left(q_{1}-q_{2}\right)$, the product of the coefficient of friction and the degree of poroelastic mismatch. For negative $f\left(q_{1}-q_{2}\right)$, the root corresponding to the Rayleigh wave moving in the opposite direction enters the unstable half plane (the response is consequently obtained by replacing $f\left(q_{1}-q_{2}\right)$ with $-f\left(q_{1}-q_{2}\right)$ and multiplying the phase velocity by -1$)$.

normal stress change is always smaller than the normal stress change, according to the relation $\Delta \bar{\sigma}=\left(1-q_{0}\right) \Delta \sigma$, since $q_{0}$ is positive for all values of Skempton's coefficient. Adding a contrast in poroelastic properties across the fault alters the region of stability, but only mildly.

\subsubsection{Limit of Identical Far-Field Elastic Properties}

[44] When far-field elastic properties are identical, then $\hat{M}_{21}=0$, and equation (31) reduces to

$$
\hat{D}_{1}=-\frac{Q}{\left(1-f \hat{L}_{1}\right) \hat{M}_{11}},
$$

indicating that bimaterial effects persist in this limit, provided that there is a poroelastic dissimilarity. If the poroelastic properties are also identical, then $\hat{L}_{1}=0$.

[45] For identical elastic materials,

$$
\hat{M}_{11}=-\frac{\mu|k|}{2} \frac{R}{\alpha_{s}\left(1-\alpha_{s}^{2}\right)},
$$

which vanishes at zero and at the Rayleigh speed. The other pole in the slip response occurs when $1-f \hat{L}_{1}=0$. In the limit of elastic similarity, $\hat{L}_{1}=\left(q_{1}-q_{2}\right) \hat{J}_{1}$, such that the stability equation is

$$
|k| R=-i f\left(q_{1}-q_{2}\right) k \alpha_{s}\left(1-\alpha_{s}^{2}\right) .
$$

Now define $w=f\left(q_{1}-q_{2}\right)$. When $w \rightarrow 0$, then this has as a solution the Rayleigh speed. Denote this solution $S_{0}= \pm i c_{R}$, where $c_{R}$ is the Rayleigh speed. For small $w$, expand the root as $S=S_{0}+w S_{1}+O\left(w^{2}\right)$, and identify

$$
S_{1}=-\left.\frac{i k \alpha_{s}\left(1-\alpha_{s}^{2}\right)}{|k| R^{\prime}}\right|_{S=S_{0}},
$$

which is real and satisfies $S_{1}\left( \pm i c_{R}\right)= \pm S_{1}\left(i c_{R}\right)$. Thus the term $w S_{1}$ is also real, such that the roots $S$ move in opposite directions from $S_{0}= \pm i c_{R}$ parallel to the real axis, one into the unstable half plane and the other into the stable half plane.

[46] Sliding is consequently always ill-posed for arbitrarily small $w=f\left(q_{1}-q_{2}\right)$ since the Rayleigh wave always exists. The growth rate (which approaches zero as $w \rightarrow 0$ ) and phase velocity of this root are shown in Figure 6. Note that this instability exists even if the elastic moduli within the damage fringes have such extreme contrasts that no generalized Rayleigh wave exists for those parameters. As $w$ increases from zero, the Rayleigh wave becomes unstable and its phase velocity increases. After reaching a maximum growth rate at $w \approx 1$, the growth rate decreases with increasing $m$. In the limit that $w \rightarrow \infty$, sliding becomes stable and the phase velocity of the mode approaches the shear wave speed.

\section{Spontaneous Ruptures With Regularized Slip-Weakening Coulomb Friction}

[47] We have also implemented the poroelastic fault zone model into a spontaneous rupture code, which enables us to explore the effects of poroelastic mismatch beyond the constraints of the linear stability analysis. In particular, the stability analysis of the previous section suggests that, in the case of opposing poroelastic and elastic bimaterial effects, a wave speed contrast of greater than $\sim 5 \%$ is necessary to counteract the poroelastic bimaterial effect with a factor-often permeability contrast (Figure 4). More generally, we are interested in exploring how the elastic and poroelastic mismatch effects interact with one another. One objective is to determine the range of parameters for which one effect dominates the other. For simplicity in this initial study, and for consistency with several previous studies, we address these issues using a slip-weakening friction law (appropriately regularized, as described below). We emphasize that this approach is not likely to reveal a host of potentially relevant bimaterial effects that might arise when considering more realistic friction laws (e.g., the pulse-like rupture mode [Ampuero and Ben-Zion, 2008]), but does suffice for the purposes described above.

[48] The analysis of the previous section shows that for parameter values of interest to us (wave speed contrasts of less than $30 \%$ ), sliding is unstable at any constant $f$. Moreover, sliding at constant $f$ is not only unstable, but it is also ill-posed since the growth rate of the instability diverges as the wavelength of the perturbation goes to zero. Numerical solutions with an unregularized friction law will not converge. Ranjith and Rice [2001] showed that the response is regularized if, instead of evaluating the fault strength as $\tau=f \bar{\sigma}$, the strength evolves toward this value over some finite slip or timescale. This regularization procedure does not stabilize any modes; it instead causes the otherwise diverging growth rate of the shortest wavelengths to peak at a wavelength proportional to the regularizing slip or timescale, and then to approach zero as the wavelength approaches zero. Cochard and Rice [2000] confirmed that this regularization procedure allows for convergent numerical solutions.

\subsection{Numerical Implementation of Poroelastic Response}

[49] We use the spectral boundary integral equation method in the form that treats both half-spaces indepen- 


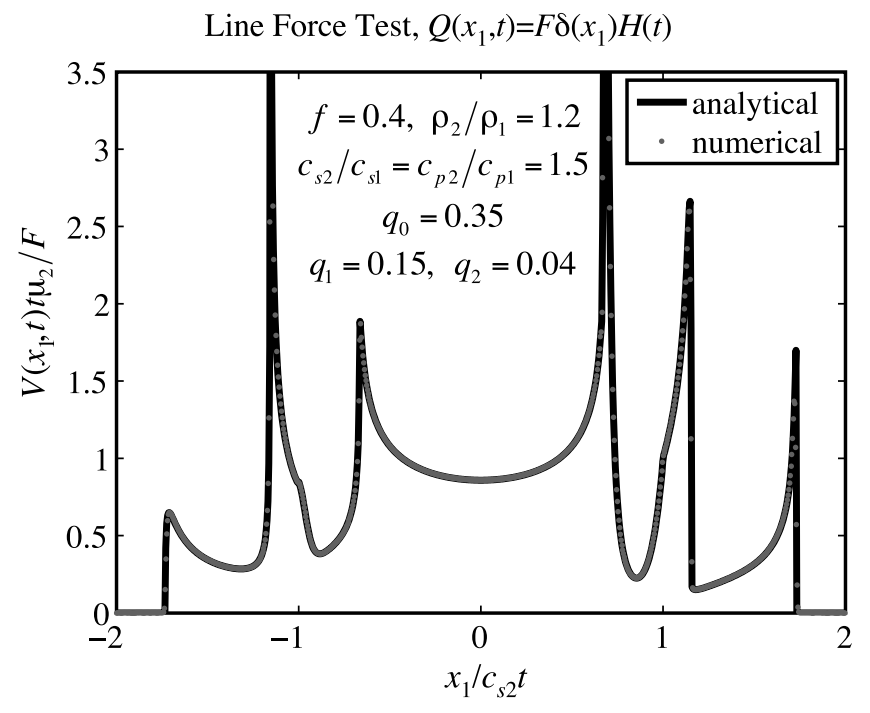

Figure 7. Validation of the numerical method against a self-similar analytical solution for the slip velocity response to the step function application of a line force on an interface that slides at a constant coefficient of friction. Parameters lie within the stable region of parameter space, so no regularization is needed.

dently before coupling them with frictional interface conditions [Breitenfeld and Geubelle, 1998; Cochard and Rice, 2000]. The important addition to existing methods required to include the poroelastic effect is evaluation of pore pressure. As equation (9) indicates, this requires knowledge of the normal stress $\sigma_{22}$ on the fault (which is available in the method) and the fault parallel strain $\epsilon_{11}$ (and also $\epsilon_{33}$ for the general three-dimensional problem). The spectral method provides an easy route toward evaluating $\epsilon_{11}^{ \pm}\left(x_{1}, t\right)=$ $\partial u_{1}^{ \pm}\left(x_{1}, t\right) / \partial x_{1}$ by multiplication of the Fourier coefficients of the displacements (which are also available in the method) by $i k$ and subsequent inversion of the transforms.

[50] The numerical method is validated by comparison to an analytical solution, similar to that presented by Harris and Day [1997], for the response of a sliding interface to a line force: $Q\left(x_{1}, t\right)=F \delta\left(x_{1}\right) H(t)$, where $\delta(\cdot)$ and $H(\cdot)$ are the Dirac delta function and Heaviside step function, respectively. The response is readily obtained from equation (31) by taking a superposition of Fourier modes using the appropriate $\hat{Q}$ for each mode. The transforms are inverted by the Cagniard-de Hoop method. Details of the method, which requires numerical integration, are given by Dunham [2007]. A comparison of the results is shown in Figure 7. Note that we have selected parameters from the stable region of parameter space, so that our numerical calculation in this case requires no regularization.

\subsection{Slip-Weakening Ruptures}

[51] For our spontaneous rupture simulations, we employ a time-regularized friction law of the form

$$
\frac{d \tau}{d t}=-\frac{1}{t^{*}}[\tau-f(\sigma-p)]
$$

in which $t^{*}$ is the regularizing timescale over which $\tau$ evolves toward $f(\sigma-p)$ and $f$ is the coefficient of friction.
The friction coefficient depends on slip according to the linear slip-weakening law [Ida, 1972; Andrews, 1976]:

$$
f_{\mathrm{sw}}(\delta)=f_{s}-\left(f_{s}-f_{d}\right) \min \left\{\delta, D_{c}\right\} / D_{c},
$$

in combination with forced expansion for nucleation purposes [Andrews, 1985]:

$$
f_{\mathrm{e}}\left(x_{1}, t\right)=\max \left\{f_{d}+A\left(\left|x_{1}\right|-v_{\mathrm{e}} t\right), f_{d}\right\} .
$$

The friction coefficient used in equation (39) is taken as the smaller of the two:

$$
f=\min \left\{f_{\mathrm{sw}}, f_{\mathrm{e}}\right\} .
$$

This method forces an expansion of the rupture at constant speed $v_{\mathrm{e}}$ during the initial portion of the simulation (when slip is sufficiently small that $f_{\mathrm{sw}}>f_{\mathrm{e}}$ ). As the rupture expands, the rate of weakening of friction with slip at the crack tips increases. This increase occurs until the weakening rate at a particular crack tip reaches the weakening rate associated with the slip-weakening law, at which time the slip-weakening process takes over $\left(f_{\mathrm{sw}}<f_{\mathrm{e}}\right)$ and the rupture propagates spontaneously. This is illustrated in Figure 8 for the case of identical elastic and poroelastic materials. In the limit that $v_{\mathrm{e}} \rightarrow 0$, this nucleation procedure is equivalent to quasi-statically expanding an initial crack on the fault until it becomes unstable. It is important to note

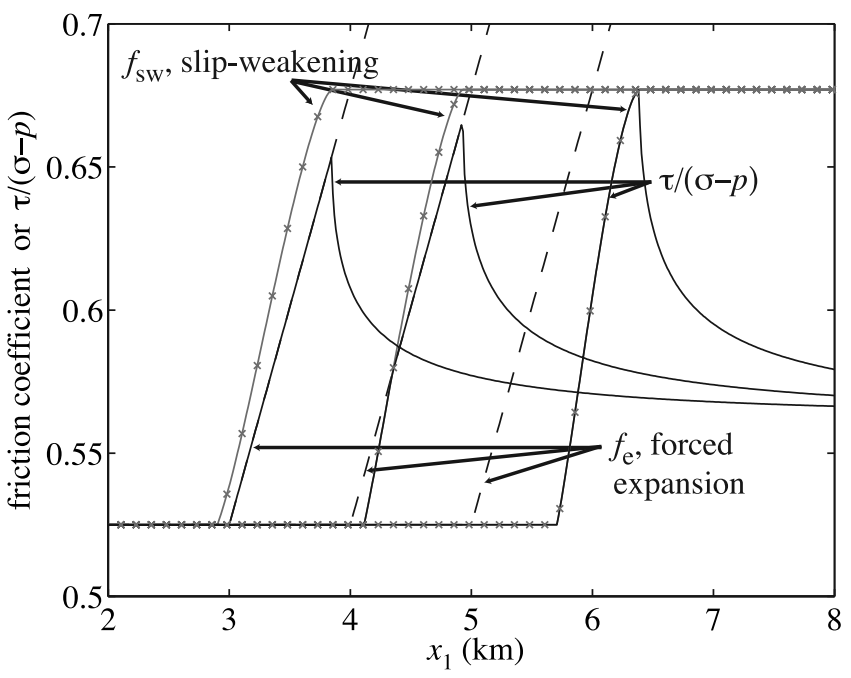

Figure 8. Nucleation of spontaneous ruptures by initially forcing expansion at constant speed, illustrated by snapshots of normalized shear stress, $\tau /(\sigma-p)$, (solid black lines) just prior to, during, and after the transition to spontaneous propagation. Early in the simulation, weakening at the rupture front is forced by a moving pattern of fault strength set by a friction coefficient, $f_{\mathrm{e}}$, that increases linearly with distance along the fault from a point that expands at a constant speed (dashed lines). Later in the simulation, the fault strength is determined by a slip-weakening friction coefficient, $f_{\text {sw }}$ (gray lines marked with cross). By setting the fault strength equal to the minimum of $f_{\mathrm{e}}$ and $f_{\mathrm{sw}}$ times the effective normal stress, the rupture smoothly transitions from forced expansion to spontaneous propagation. 


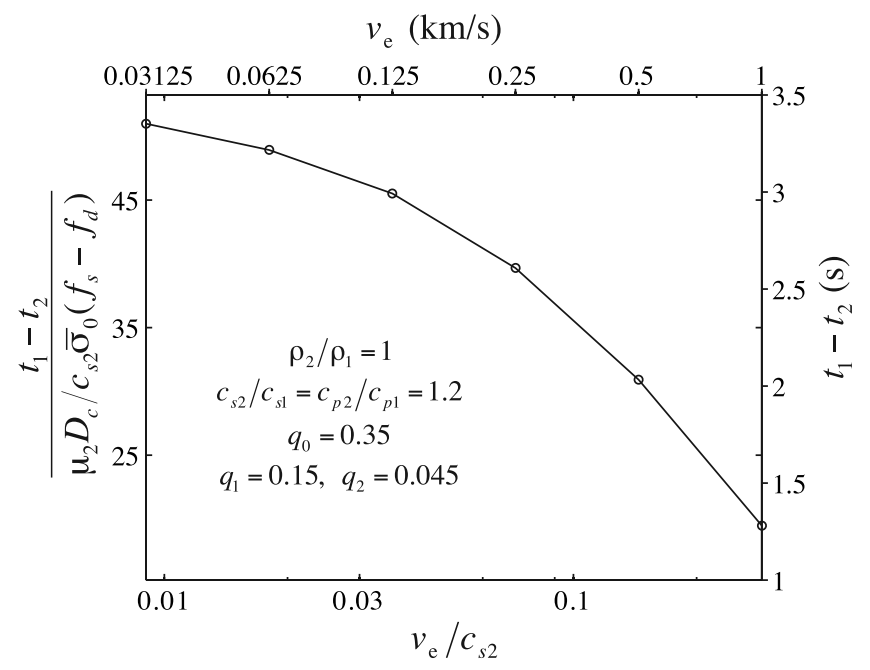

Figure 9. Difference in time at which the two crack tips become unstable as a function of forced crack expansion speed, $v_{\mathrm{e}}$. No divergence as $v_{\mathrm{e}} \rightarrow 0$ is observed, so quasistatic nucleation is expected to generate bilaterally propagating ruptures.

that for dissimilar materials the transition to spontaneous propagation occurs earlier for one crack tip than the other since the effective normal stress changes are of opposite sign for the two tips.

[52] While our simulations are conducted using nondimensional parameters (implying that they are valid at any scale of interest), we also provide dimensional values using the parameters given in Table 2. These parameters are similar to those used in the code validation tests of the Southern California Earthquake Center (SCEC) [Harris and Archuleta, 2004], but with the initial stress level decreased to prevent ruptures from exceeding the shear wave speed (of either side of the fault). The parameters, if used in threedimensional simulations, are appropriate for modeling earthquakes with magnitudes $\sim 6-7$. We use them despite their unrealistic feature for slip on what is considered to be, effectively, a mathematical plane: temperature changes, ignored in the SCEC exercise, would then be so large that melting would have to occur for realistic amounts of slip in significant events. Our results are presented in both dimensional and nondimensional forms, with the stress scale in the latter taken as $\bar{\sigma}_{0}\left(f_{s}-f_{d}\right)$; this quantity corresponds to the strength drop in the absence of effective normal stress changes. The slip-weakening law provides a displacement scale in the slip-weakening distance, $D_{c}$. The distance scale, $\mu_{2} D_{c} / \bar{\sigma}_{0}\left(f_{s}-f_{d}\right)$, is proportional to the critical nucleation zone size under linear slip-weakening friction in the absence of effective normal stress changes [Uenishi and Rice, 2003]. The scale is also identical, apart from a dimensionless prefactor of $9 \pi / 32(1-v)$ (which is $\approx 1.2$ for $v=1 / 4)$, to $R_{0}^{*}$, defined by Rice et al. [2005] as the extent of the slipweakening zone for quasi-static propagation of a slip pulse. The wave speed scale is $c_{s 2}$, and the (particle and slip) velocity scale is $c_{s 2} \bar{\sigma}_{0}\left(f_{s}-f_{d}\right) / \mu_{2}$. The timescale is $\mu_{2} D_{c} / \bar{\sigma}_{0}$ $\left(f_{s}-f_{d}\right) c_{s 2}$, which measures how long it takes for a slip equal to $D_{c}$ to occur when sliding at the slip velocity scale.
Wave speeds are decreased on the $x_{2}>0$ side with the values on the other side held fixed. To obtain sufficient numerical resolution of the rupture fronts, a grid spacing of $h=12.5 \mathrm{~m}$ is used (which places 56.2 points within the distance scale). The regularizing timescale is chosen to be $t^{*}$ $=0.01 \mathrm{~s}$, which is 20.7 times shorter than the characteristic timescale; this provides a distinct separation of the two evolution processes.

[53] We study ruptures with six parameter sets. The first four $(1 \mathrm{a}-1 \mathrm{~d})$ are for the case of opposing poroelastic and elastic effects, as marked in the left panel of Figure 4. In particular, there is no density contrast, a poroelastic mismatch consistent with a factor-of-ten permeability contrast, and four wave speed contrasts $(0 \%, 10 \%, 20 \%, 30 \%)$. The last two parameter sets ( $2 \mathrm{a}$ and $2 \mathrm{~b}$ ) are for enhancing effects, as shown in the right panel of Figure 4. Again, there is no density contrast, a poroelastic mismatch consistent with a factor-of-ten permeability contrast, and wave speed contrasts of $10 \%$ and $20 \%$.

[54] We first study how the difference in nucleation times for the two crack tips depends on $v_{\mathrm{e}}$ for one of the extreme limits (reinforcing poroelastic and elastic mismatch effects with a wave speed contrast of $20 \%$ ). This is shown in Figure 9. As $v_{\mathrm{e}}$ decreases, the difference in nucleation times for the two crack tips increases, but in a manner that suggests a finite time difference in the limit that $v_{\mathrm{e}} \rightarrow 0$. In particular, no divergence in this limit is observed; had a divergence been observed, it would have indicated purely unilateral propagation after nucleation. A compromise between computational efficiency (which favors larger $v_{\mathrm{e}}$ ) and the desired quasi-static nucleation is obtained by using $v_{\mathrm{e}}=$ $0.5 \mathrm{~km} / \mathrm{s}=0.144 c_{s 2}$. In all cases, we set the rate of increase of friction with distance in the forced nucleation procedure to $A=0.152 \mathrm{~km}^{-1}=0.703 \bar{\sigma}_{0}\left(f_{s}-f_{d}\right)^{2} / \mu_{2} D_{c}$. This value was chosen such that a drop in friction coefficient equal to $f_{s}-f_{d}=0.152$ occurs over $1 \mathrm{~km}$, which is roughly equal to the distance scale $\mu_{2} D_{c} / \bar{\sigma}_{0}\left(f_{s}-f_{d}\right)$.

[55] The results of our spontaneous rupture simulations are presented in Figures 10-14. On the whole, the ruptures behave as might have been predicted by the linear stability analysis. While the ruptures take the form of expanding cracks with this friction law, there is asymmetry in the rupture process, with rupture speeds and slip rates being higher in the direction in which the effective normal stress is reduced.

[56] In contrast to the slip and slip velocity fields, there is considerable complexity in the stress and pore pressure fields. By far the easiest case to understand is Ex. 1a (poroelastic mismatch, but no far-field elastic mismatch). As seen in Figure 14, pore pressure changes only when the fault is slipping. This is consistent with our expression for the pore pressure change given in equation (9) since, in the absence of an elastic mismatch, normal stress on the fault remains constant. The only contribution to pore pressure comes from the fault parallel strains, which are equal in magnitude and opposite in sign across the fault. The pore pressure change can consequently be written (assuming plane strain conditions) in terms of the slip gradient as $\Delta p=-(1 / 2)\left(q_{1}-q_{2}\right) \mu d \delta / d x_{1}$; in the absence of slip, the pore pressure remains unaltered. The change in pore pressure is the only contribution to the effective normal stress 

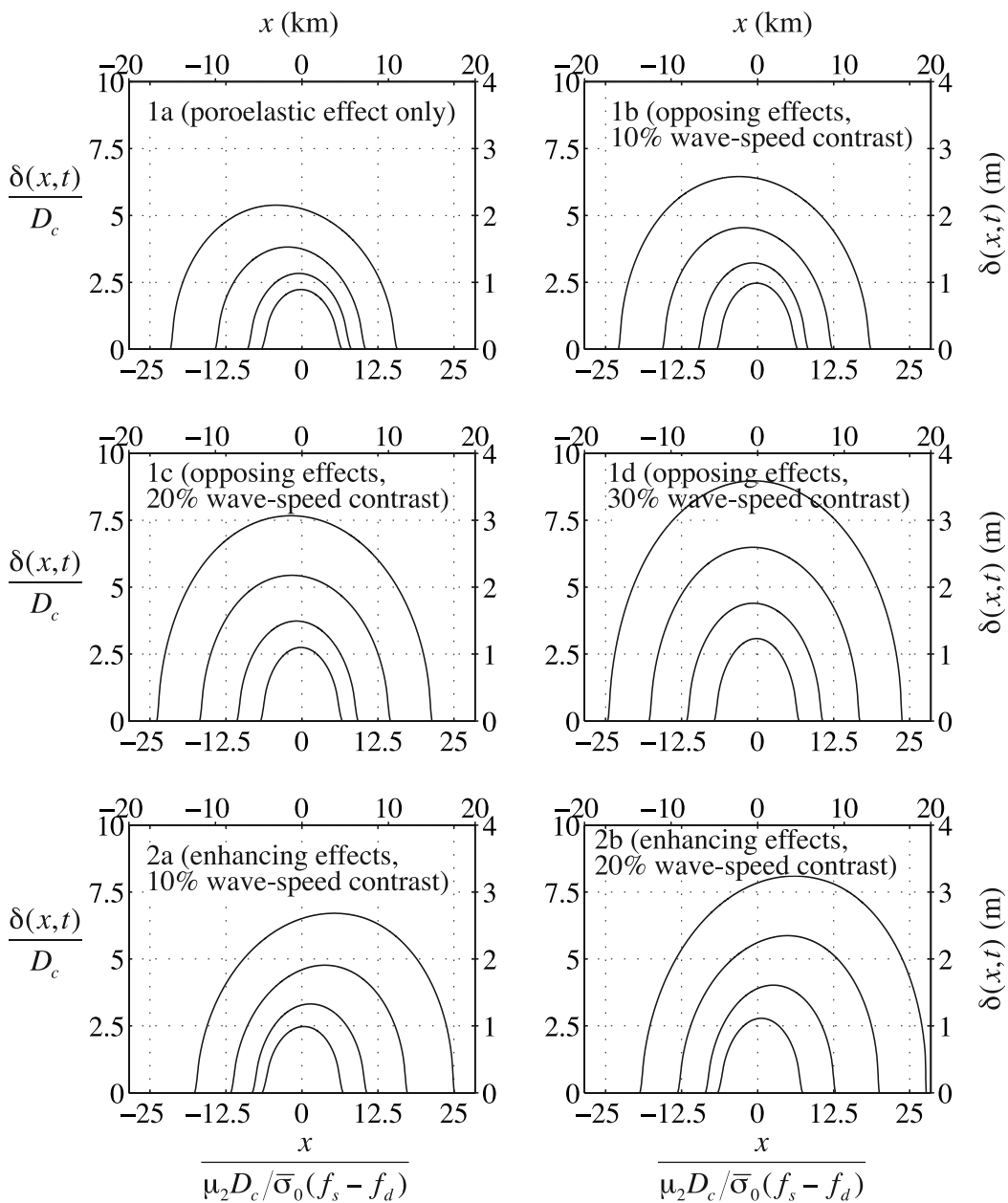

Figure 10. Snapshots of slip every $2 \mathrm{~s}\left(=9.86 \mu_{2} D_{\mathrm{c}} / \bar{\sigma}_{0}\left(f_{s}-f_{d}\right) c_{s 2}\right)$ for the six cases $(1 \mathrm{a}-1 \mathrm{~d}, 2 \mathrm{a}$, and $2 \mathrm{~b})$ defined in Figure 4. Both nondimensional (bottom, left) and dimensional (top, right) scales are provided.

change, so the distribution of pore pressure appears also in the plot of effective normal stress (Figure 13), but with the sign reversed according to our convention. The effective normal stress change influences the shear stress field (Figure 12) by slightly altering the strength drop.

[57] Next, consider a mismatch in far-field elastic properties. For ruptures moving less than the generalized Rayleigh speed, the normal stress is reduced behind the rupture front propagating in the direction of displacement of the more compliant medium (and increased at the opposite front). The sign of the normal stress change ahead of the rupture front is opposite this, however. This is discussed, for example, in the study of Rubin and Ampuero [2007] and can be seen in our simulations with large elastic mismatches and no competing poroelastic effects (e.g., the bottom panels of Figure 13). In this case, the peak and residual strengths are altered in opposite directions (Figure 12). In Ex. 2b, for example, the strength drop at the right tip is enhanced by both an increase in peak strength and a decrease in residual strength (the opposite occurs at the left tip).

[58] The steady state analyses of Weertman [1980] and Rudnicki and Rice [2006] predict that effective normal stress changes are proportional to the local value of the slip gradient, $\partial \delta / \partial x_{1}$. It follows that effective normal stress is altered only within the rupture, and not ahead of or behind it. Interestingly, our numerical simulations, as discussed in the previous paragraph, reveal substantial changes in effective normal stress outside the slipping region. Our bilaterally expanding ruptures differ from steady state slip pulses, and it is likely the expansion process, which excites a spectrum of waves with a variety of phase speeds along the fault, that is responsible for changing the effective normal stress outside of the crack. This major difference from the steady state prediction highlights the complementary nature of our numerical simulations to simple analytical models.

[59] When the poroelastic and elastic mismatch effects oppose one another, then the alterations of pore pressure and effective normal stress can be quite complex. In their model of steadily propagating slip pulses, Rudnicki and Rice [2006] found that, in the case of opposing effects, there exists a critical propagation speed below which the poroelastic effect dominates and above which the elastic mismatch effect determines the sign of the effective normal stress change. This critical speed exists only if the far-field wave speed contrast exceeds about $5-10 \%$ (the specific 

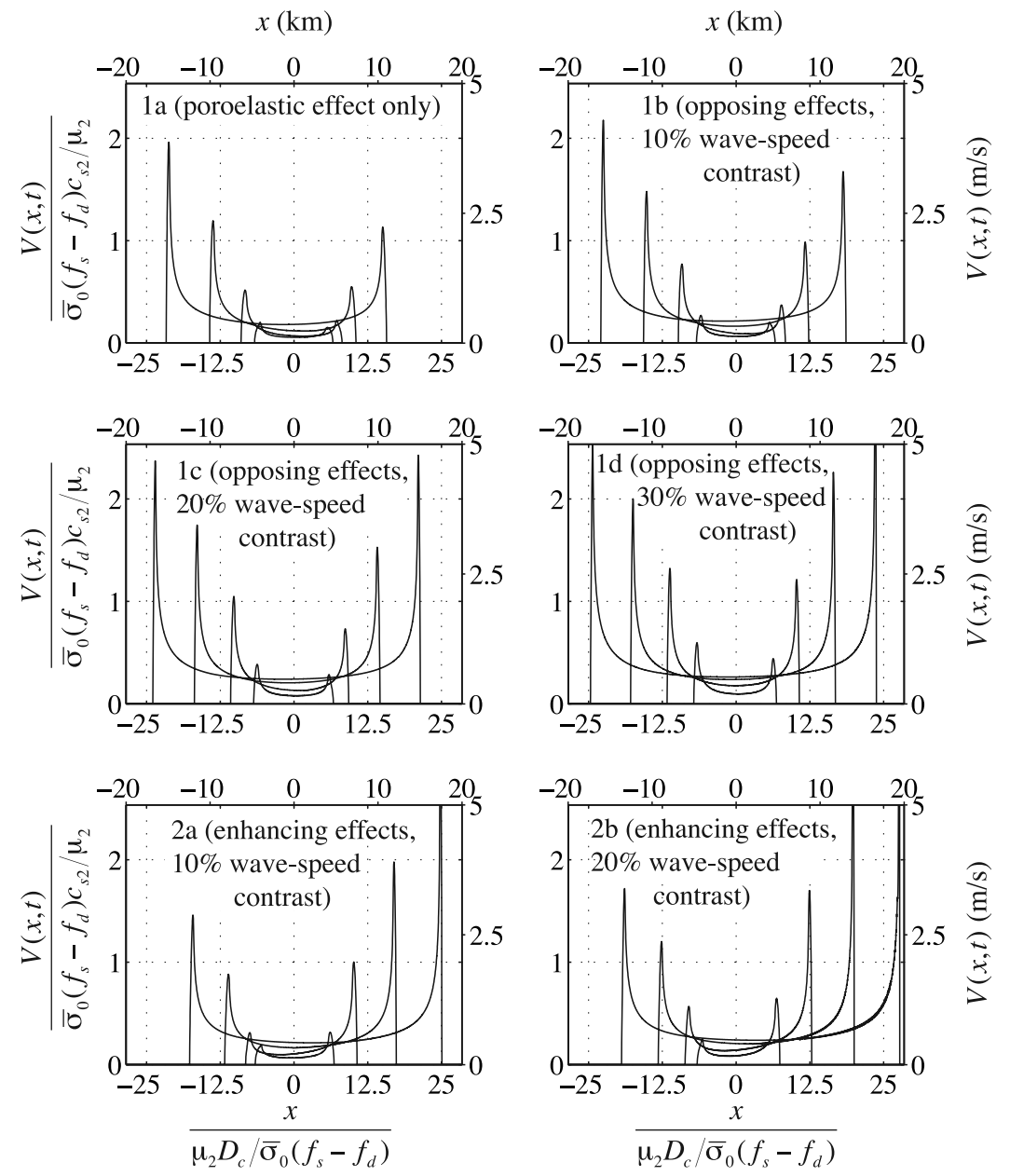

Figure 11. Same as Figure 10, but for slip velocity.

value depends on the poroelastic mismatch and Skempton's coefficient); for smaller contrasts, the poroelastic effect determines the sign of effective normal stress change at all speeds. By evaluating the expressions given by Rudnicki and Rice [2006], we can determine the rupture speed at which the sign reversal occurs, as well as the minimum elastic contrast required for elastic bimaterial effects to dominate at high speeds. This is shown in Figure 15.

[60] The numerical simulations can be interpreted in the context of the predictions of Rudnicki and Rice [2006]. At low speeds, the poroelastic bimaterial effect determines the sign of effective normal stress change at the rupture front. As the rupture accelerates, the sign of the change will reverse, but only if the elastic mismatch is sufficiently large. Figure 15 indicates that this reversal should occur in Ex. $1 \mathrm{~b}-1 \mathrm{~d}$. Consider the pore pressure distribution for Ex. 1b (opposing effects, $10 \%$ wave speed contrast). It is quite similar to that in Ex. 1a, with the additional feature of a nonzero pore pressure change ahead of the rupture fronts. As discussed following equation (9), elastic dissimilarity will induce both normal stress changes and fault-parallel strains ahead of the rupture front; these alter the pore pressure on the fault. The sign of the induced pore pressure change reverses across the rupture front, as does the sign of normal stress change, and these two fields contribute with opposing signs to the effective normal stress change. The magnitude of the pore pressure change is larger than that of the normal stress change ahead of the rupture front, while the opposite is true behind it. This leads to an effective normal stress change distribution that retains the same sign across the rupture front (and with the sign determined by the pore pressure change). Because of this, both the peak and residual strengths are altered in the same direction. The observation that the sign of effective normal stress change is set by the poroelastic effect indicates that the rupture speed is less than the critical value from Figure 15 at all times that are plotted here. Extending the simulation time beyond that shown here does indeed reveal the predicted sign reversal.

[61] With the larger elastic mismatch of Ex. 1c (opposing effects, $20 \%$ wave speed contrast), the sign reversal occurs within the simulated time; this is illustrated in Figure 16. At early times in this simulation, the situation is the same as that described above for Ex. $1 b$ (i.e., the sign of the effective normal stress change is determined by the sign of the pore pressure change behind the rupture front and, ahead of it, by the sign of the normal stress change). After accelerating above the critical rupture velocity, the normal stress change exceeds that of the pore pressure change both ahead of and behind the rupture front. This generates the reversal in the sign of effective normal stress change across the rupture 

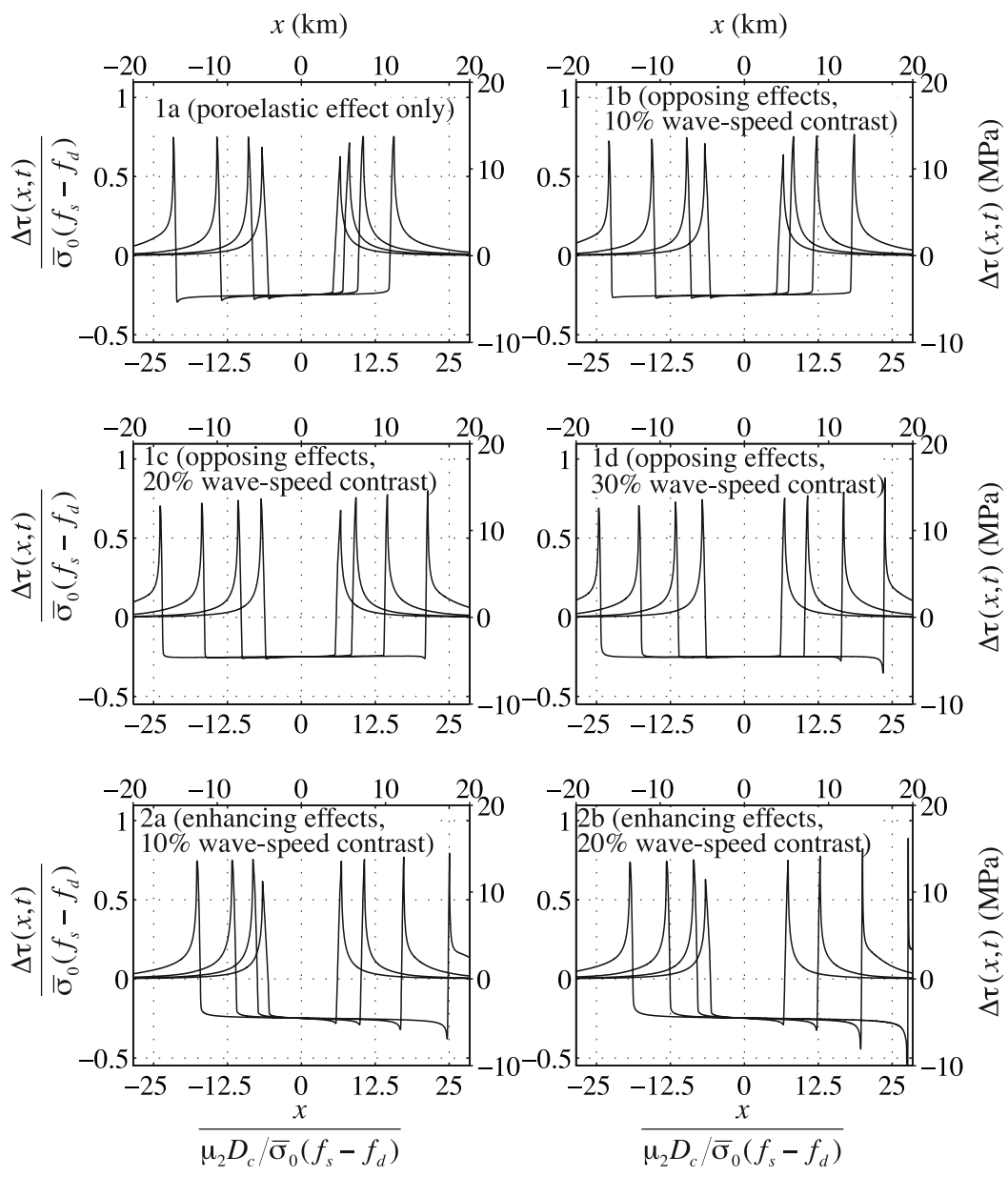

Figure 12. Same as Figure 10, but for shear stress change.

front that is evident in Figure 13. This reversal occurs for higher wave speed contrasts as well (Ex. 1d).

[62] An important prediction from the model of Rudnicki and Rice [2006] is that below a certain elastic mismatch level, the poroelastic effect will always dominate, no matter the rupture speed. To confirm this, we measured the distance, $L_{\text {reversal }}$, that the crack tip moving in the $+x_{1}$ direction must propagate before the sign reversal occurs. Figure 15 suggests that $L_{\text {reversal }}$ will diverge as the wave speed contrast approaches $4 \%$ from above. This is indeed what we find, as shown in Figure 17.

[63] Except in the most extreme case of enhancing poroelastic and elastic bimaterial effects (Ex. 1d), the effective normal stress change is a small fraction of the absolute level of effective normal stress for the parameters studied. We caution that this should not be interpreted as a universal feature of bimaterial ruptures. The amplitude of the effective normal stress change increases with propagation distance, and it appears inevitable in these calculations that after propagating sufficiently far, the effective normal stress will become tensile if one retains, unrealistically, the assumption that the fault surfaces slide but do not open. The required propagation distance for this to occur depends on both the level of material contrast and the ratio of the regularization timescale, $t^{*}$, to the timescale characterizing the slip-weakening response, $\mu_{2} D_{c} / \bar{\sigma}_{0}\left(f_{s}-f_{d}\right) c_{s 2}$. We leave an exploration of this phenomenon to later work employing a more realistic friction law and regularization procedure.

\section{Discussion}

[64] While the diffusion of pore fluids over the duration of an earthquake is negligible at most scales of interest, it plays an important role in determining the pore pressure distribution within narrow fault-bordering fringes within the zone of damaged (densely cracked, granulated) material hosting the fault slip surface. Since the shear strength of faults is proportional to the effective normal stress on them, reductions in effective normal stress (via decreases in normal stress or increases in pore pressure) weaken the fault. It has previously been noted that spatially nonuniform slip between dissimilar elastic solids alters the normal stress on faults. In this work, we explored the poroelastic fault zone model developed by Rudnicki and Rice [2006], and confirmed that a mismatch in poroelastic properties (e.g., permeability) across faults leads to a similar response. The elastic and poroelastic responses can independently cause either reductions of or increases in effective normal stress, depending on which side of the fault is more compliant and/ or more permeable. The two effects enhance each other if the compliant side is more permeable, and oppose each other if the stiff side is more permeable. The two effects 

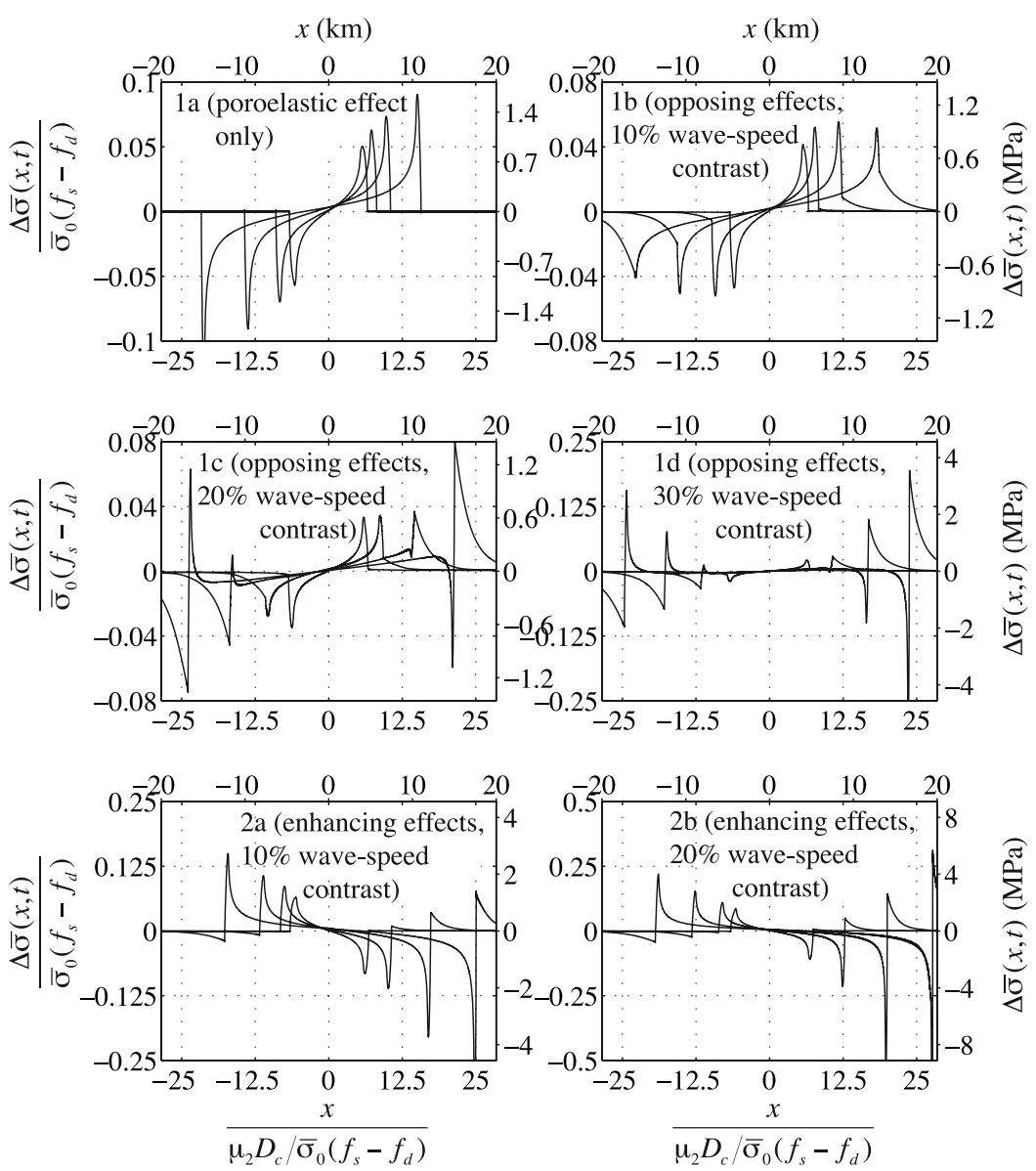

Figure 13. Same as Figure 10, but for effective normal stress change (positive in compression). 

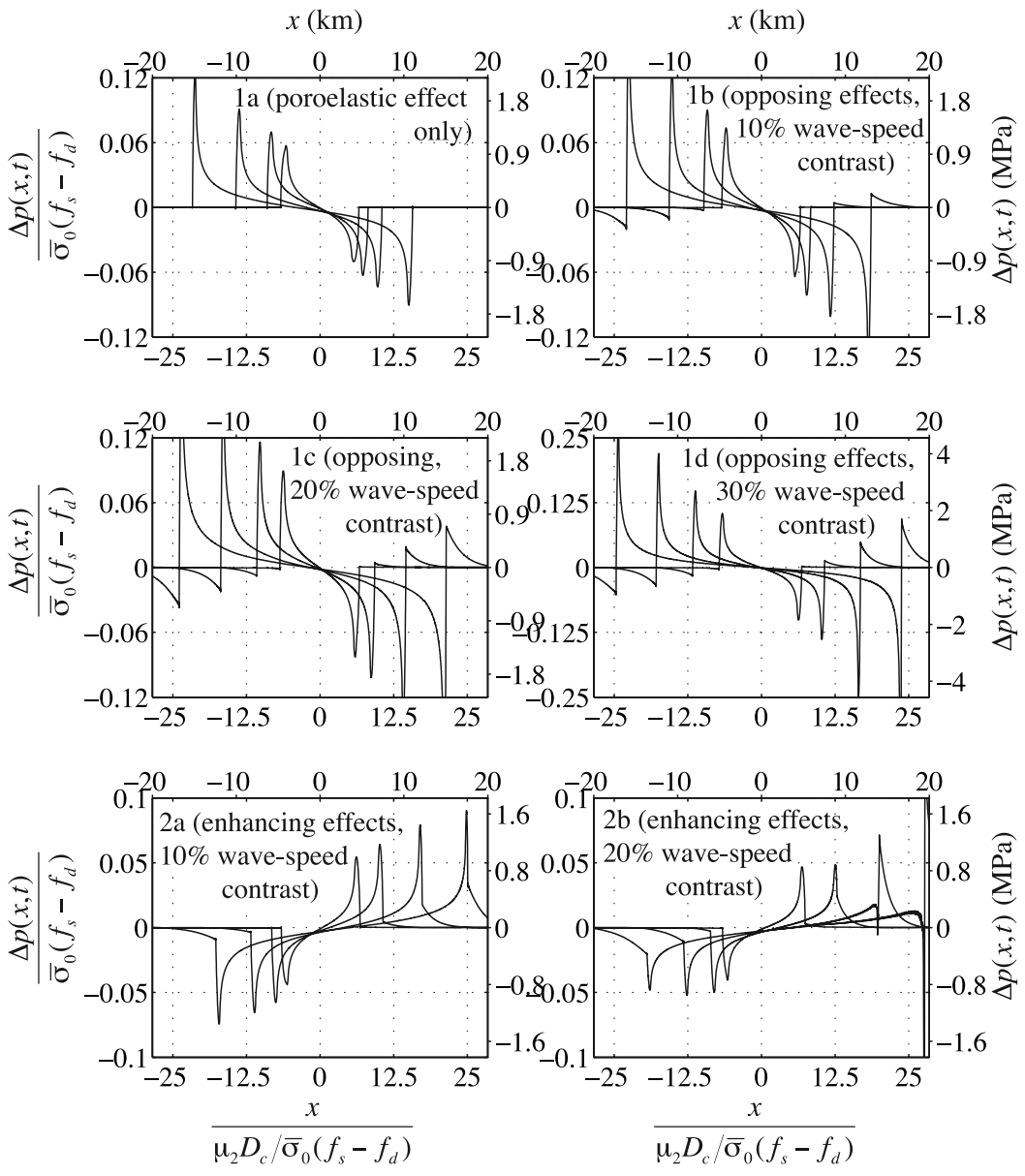

Figure 14. Same as Figure 10, but for pore pressure change. 


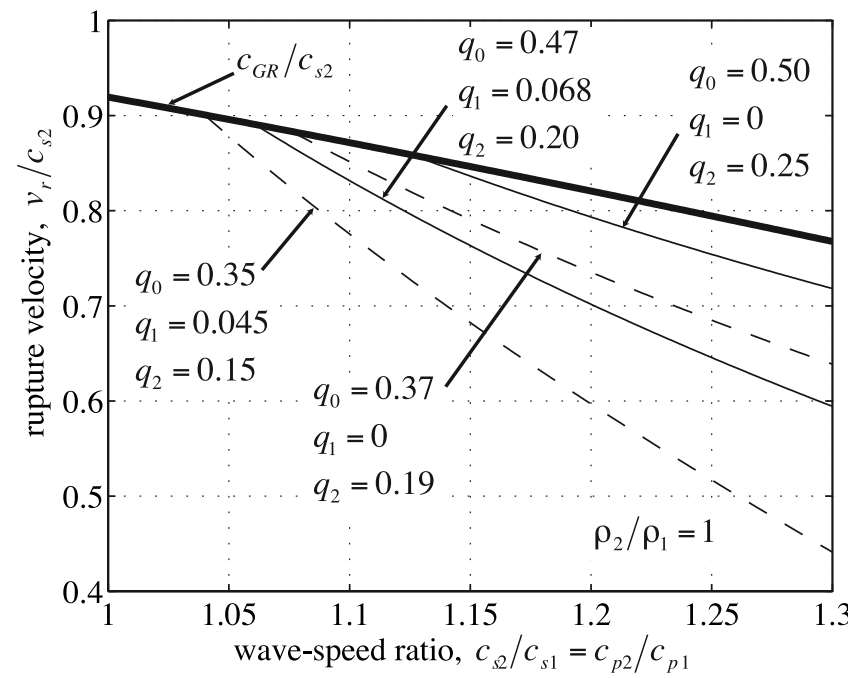

Figure 15. Critical rupture velocity for sign reversal of effective normal stress change, $\Delta \bar{\sigma}$. Below this velocity the poroelastic effect determines the sign of $\Delta \bar{\sigma}$ and above it the elastic bimaterial effect does. Also shown is the generalized Rayleigh speed, $c_{\mathrm{GR}}$, the maximum subshear rupture velocity. The critical rupture velocity is shown for several parameter sets. The lower dashed line corresponds to the poroelastic parameters used in both the stability analysis and numerical simulations (a factor-of-ten permeability contrast and Skempton's coefficients of $B_{1}=0.4$ and $B_{2}=$ 0.6 ). The upper dashed line (with $q_{1}=0$ ) is for the same parameters, but with an infinite permeability contrast. The solid lines are similar to the dashed lines, but use the larger values of Skempton's coefficients $\left(B_{1}=0.6\right.$ and $B_{2}=0.8$ ) that were estimated by E. Templeton (personal communication, 2007) for ultracataclastic fault gouge from data compiled by Rice [2006].

have comparable magnitudes for parameters representative of natural faults (a wave speed contrast of about 5-10\% and a factor-of-ten permeability contrast). Steady sliding of two elastically identical half-spaces at any constant friction

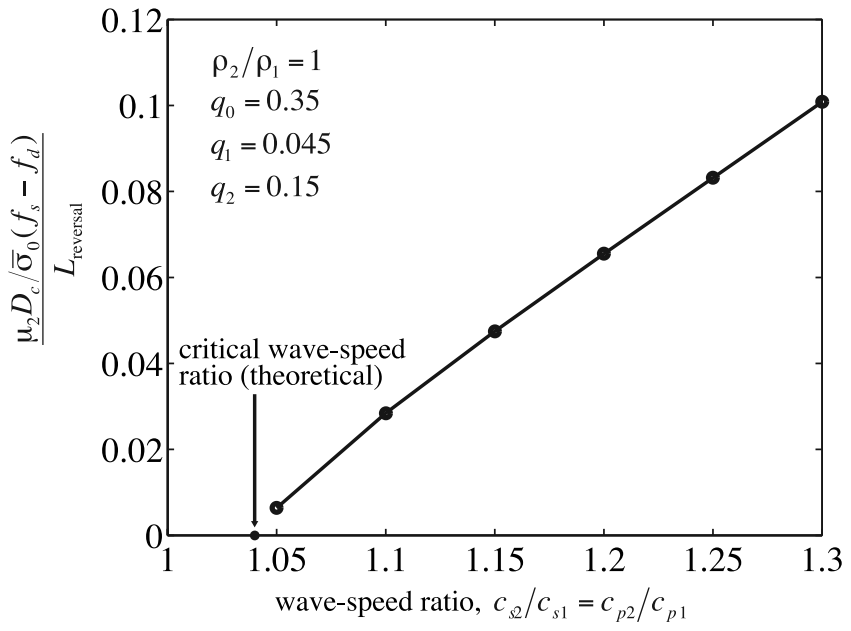

Figure 17. Divergence of the propagation distance required for a reversal in the sign of effective normal stress change at the rupture front, for the case of opposing poroelastic and elastic bimaterial effects. The numerical results are consistent with the theoretically predicted critical wave speed contrast of $4 \%$.

coefficient is unstable to perturbations if a contrast in poroelastic properties exists across the slip surface. When both elastic and poroelastic properties are discontinuous across the fault, sliding is stable only within a region of parameter space centered about a wave speed contrast of $50-100 \%$ and for friction coefficients less than a value of order unity; otherwise it is unstable. In particular, for wave speed contrasts less than about $30 \%$, the generalized Rayleigh wave exists in frictionless contact and frictional sliding is unstable for arbitrarily small coefficients of friction. The growth rate of the instability vanishes as $f \rightarrow 0$, as well as in the limit of identical materials.

[65] Since the sign of effective normal stress change is different on either side of a patch of slip, these bimaterial effects introduce asymmetry into the rupture process. Spontaneous rupture simulations employing a time-regularized slip-weakening friction law reveal bilaterally propagating
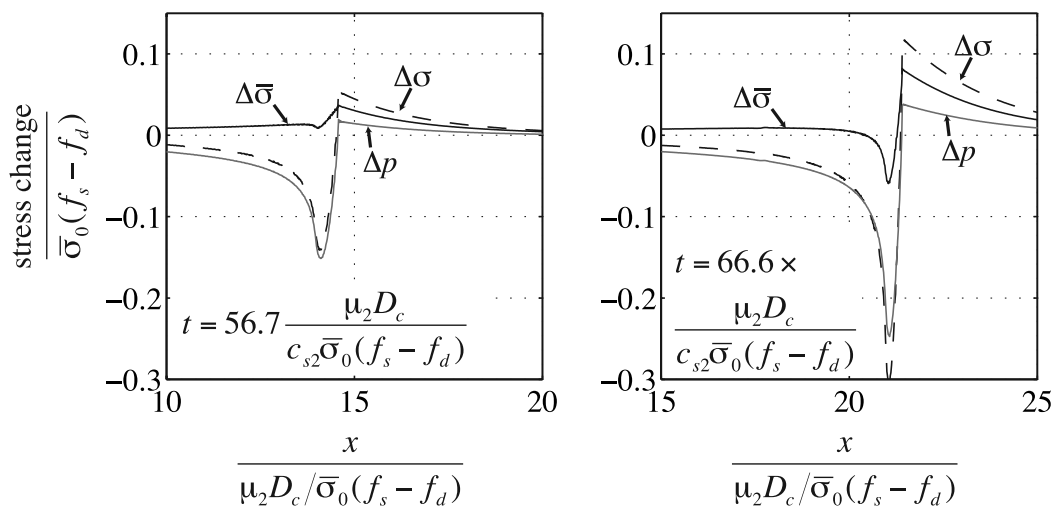

Figure 16. Sign reversal of effective normal stress change, $\Delta \bar{\sigma}$, as the rupture accelerates, from the sign determined by the poroelastic mismatch to that determined by the far-field elastic match. Shown for Ex. 1c (opposing effects, $20 \%$ wave speed contrast). The effective normal stress (positive in compression) is the difference between normal stress (positive in compression) and pore pressure (positive in tension): $\bar{\sigma}=\sigma-p$. 
crack-like ruptures, but rupture speeds and slip rates are larger in the direction in which effective normal stress is reduced. We caution that such results are likely sensitive to the particular friction law, and that a variety of interesting and important phenomena might result when coupling the bimaterial response with friction laws more realistic than the slip-weakening law used here. Further investigation of this issue is a high priority, and the study of Ampuero and Ben-Zion [2008] using a slip- and velocity-weakening friction law reveals a variety of rupture modes (including unilateral pulses). However, the regularized slip-weakening law we employ suffices to demonstrate that the poroelastic effect we study is just as important as that due to a far-field elastic mismatch. This implies that rupture propagation direction cannot be predicted solely by measuring the elastic mismatch across a fault. Geologic observations are needed to determine if there is a systematic tendency for the slip surface to be located on one side or the other of the fault core.

[66] A further issue in evaluating the poroelastic response is that hydraulic diffusivity is likely to be altered by dynamically induced off-fault inelastic stresses near the rupture front. The stress state depends on rupture propagation direction and prestress angle [Poliakov et al., 2002; Rice et al., 2005]. In many circumstances, the prestress direction favors inelastic response on the extensional side of the rupture. Assuming that this response increases hydraulic diffusivity (e.g., by opening fluid flow paths that were sealed during the interseismic period) and that this dynamically induced poroelastic contrast dominates any preexisting contrasts, then the poroelastic bimaterial effect will always oppose the elastic bimaterial effect. An important, but difficult, task is to quantify changes in hydraulic diffusivity resulting from the inelastic material response.

[67] Acknowledgments. E.M. Dunham gratefully acknowledges partial support from a Reginald A. Daly Postdoctoral Fellowship at Harvard. This research was also supported by NSF-EAR grants 0510193 and 0440145 and the Southern California Earthquake Center, funded by NSF Cooperative Agreement EAR-0106924 and USGS Cooperative Agreement 02HQAG0008. The SCEC contribution number for this paper is 1124. We thank Yehuda Ben-Zion, an anonymous reviewer, and the associate editor for their insightful comments and valuable suggestions.

\section{References}

Achenbach, J. D., and H. I. Epstein (1967), Dynamic interaction of a layer and a half-space, J. Eng. Mech. Div., EM5, 27-42.

Adams, G. G. (1995), Self-excited oscillations of two elastic half-spaces sliding with a constant coefficient of friction, J. Appl. Mech., 62, 867872 .

Ampuero, J.-P., and Y. Ben-Zion (2008), Cracks, pulses and macroscopic asymmetry of dynamic rupture on a bimaterial interface with velocityweakening friction, Geophys. J. Int., 173(2), 674-692, doi:10.1111/ j.1365-246X.2008.03736.x.

Andrews, D. J. (1976), Rupture velocity of plane strain shear cracks, J. Geophys. Res., 81, 5679-5687.

Andrews, D. J. (1985), Dynamic plane-strain shear rupture with a slipweakening friction law calculated by a boundary integral method, Bull. Seismol. Soc. Am., 75(1), 1-21.

Andrews, D. J., and Y. Ben-Zion (1997), Wrinkle-like slip pulse on a fault between different materials, J. Geophys. Res., 102, 553-571.

Andrews, D. J., and R. A. Harris (2005), The wrinkle-like slip pulse is not important in earthquake dynamics, Geophys. Res. Lett., 32, L23303, doi:10.1029/2005GL023996.

Anooshehpoor, A., and J. Brune (1999), Wrinkle-like Weertman pulse at the interface between two blocks of foam rubber with different velocities, Geophys. Res. Lett., 26(13), 2025-2028.

Ben-Zion, Y. (2006a), A comment on "Material contrast does not predict earthquake rupture propagation direction" by R. A. Harris and S. M. Day, Geophys. Res. Lett., 33, L13310, doi:10.1029/2005GL025652.
Ben-Zion, Y. (2006b), A comment on "The wrinkle-like slip pulse in not important in earthquake dynamics" by D. J. Andrews and R. A. Harris, Geophys. Res. Lett., 33, L06310, doi:10.1029/2005GL025372.

Ben-Zion, Y., and D. J. Andrews (1998), Properties and implications of dynamic rupture along a material interface, Bull. Seismol. Soc. Am., 88 $1085-1094$

Ben-Zion, Y., and Y. Huang (2002), Dynamic rupture on an interface between a compliant fault zone layer and a stiffer surrounding solid, J. Geophys. Res., 107(B2), 2042, doi:10.1029/2001JB000254.

Ben-Zion, Y, and Z. Shi (2005), Dynamic rupture on a material interface with spontaneous generation of plastic strain in the bulk, Earth Plant. Sci. Lett., 236, 486-496, doi:10.1016/j.eps1.2005.03.025.

Ben-Zion, Y., Z. Peng, D. Okaya, L. Seeber, J. G. Armbruster, N. Ozer, A. J. Michael, S. Baris, and M. Aktar (2003), A shallow fault-zone structure illuminated by trapped waves in the Karadere-Duzce branch of the North Anatolian fault, western Turkey, Geophys. J. Int., 152, 699-717.

Boettcher, M. S., and C. Marone (2001), Effects of normal stress variation on the strength and stability of creeping faults, J. Geophys. Res., 109, B03406, doi:10.1029/2003JB002824.

Brace, F., and J. D. Byerlee (1966), Stick-slip as a mechanism for earthquakes, Science, 153(3739), 990-992.

Breitenfeld, M. S., and P. H. Geubelle (1998), Numerical analysis of dynamic debonding under 2D in-plane and 3D loading, Int. J. Fract., 93, 13-38.

Brietzke, G., and Y. Ben-Zion (2006), Examining tendencies of in-plane rupture to migrate to material interfaces, Geophys. J. Int., 167, 807-819, doi:10.1111/j.1365-246X.2006.03137.x.

Budiansky, B., and R. J. O'Connell (1976), Elastic moduli of a cracked solid, Int. J. Solids Struct., 12, 81-97.

Chester, F. M., and J. S. Chester (1998), Ultracataclasite structure and friction processes of the Punchbowl fault, San Andreas system, California, Tectophysics, 295, 199-221.

Chester, F. M., J. S. Chester, D. L. Kirschner, S. E. Schulz, and J. P. Evans (2004), Structure of large-displacement, strike-slip fault zones in the brittle continental crust, in Rheology and Deformation in the Lithosphere at Continental Margins, edited by G. D. Karner et al., pp. 223-260, Columbia Univ. Press, New York.

Cocco, M., and J. R. Rice (2002), Pore pressure and poroelasticity effects in Coulomb stress analysis of earthquake interactions, J. Geophys. Res., 107(B2), 2030, doi:10.1029/2000JB000138.

Cochard, A., and J. R. Rice (2000), Fault rupture between dissimilar materials: Ill-posedness, regularization and slip-pulse response, J. Geophys. Res., 105, 25,891-25,907.

Dalguer, L. A., and S. M. Day (2007), 3D effects on dynamic rupture propagation at bimaterial interface (abstract of 2007 annual meeting of the Seismological Society of America, 11 - 13 April 2007, Kona, Hawaii), Seismol. Res. Lett., 78(2), 303.

Dor, O., T. K. Rockwell, and Y. Ben-Zion (2006), Geological observations of damage asymmetry in the structure of the San Jacinto, San Andreas and Punchbowl Faults in Southern California: A possible indicator for preferred rupture propagation direction, Pure Appl. Geophys., 163(2-3), 301-349, doi:10.1007/s00024-005-0023-9.

Dunham, E. M. (2007), Conditions governing the occurrence of supershear ruptures under slip-weakening friction, J. Geophys. Res., 112, B07302, doi:10.1029/2006JB004717.

Eberhart-Phillips, D., and A. J. Michael (1998), Seismotectonics of the Loma Prieta, California, region determined from three-dimensional $v_{p}$, $v_{p} / v_{s}$, and seismicity, J. Geophys. Res., 103, 21,099-21,120.

Feng, R., and T. V. McEvilly (1983), Interpretation of seismic reflection profiling data for the structure of the San Andreas fault zone, Bull. Seismol. Soc. Am., 73, 1701-1720.

Fuis, G. S., et al. (2003), Fault systems of the 1971 San Fernando and 1994 Northridge earthquakes, southern California: Relocated aftershocks and seismic images from LARSE II, Geology, 31(2), 171-174.

Geubelle, P. H., and J. R. Rice (1995), A spectral method for three-dimensional elastodynamic fracture problems, J. Mech. Phys. Solids, 43, $1791-$ 1824

Harris, R. A., and R. J. Archuleta (2004), Seismology: Earthquake rupture dynamics: Comparing the numerical simulation methods, Eos Trans. $A G U, 85(34), 321$, doi:10.1029/2004EO340003.

Harris, R. A., and S. M. Day (1997), Effects of a low-velocity zone on a dynamic rupture, Bull. Seismol. Soc. Am., 87, 1267-1280.

Harris, R. A., and S. M. Day (2005), Material contrast does not predict earthquake rupture propagation direction, Geophys. Res. Lett., 32, L23301, doi:10.1029/2005GL023941.

Hart, D. J., and H. F. Wang (1995), Laboratory measurements of a complete set of poroelastic moduli for Berea sandstone and Indiana limestone, J. Geophys. Res., 100, 17,741-17,751.

Heermance, R., Z. K. Shipton, and J. P. Evans (2003), Fault structure control on fault slip and ground motion during the 1999 rupture of the Chelungpu Fault, Taiwan, Bull. Seismol. Soc. Am., 93(3), 1034-1050. 
Hill, R. (1962), Acceleration waves in solids, J. Mech. Phys. Solids, 10(1), 1-16, doi:10.1016/0022-5096(62)90024-8.

Hong, T., and C. Marone (2005), Effects of normal stress perturbations on the frictional properties of simulated faults, Geochem. Geophys. Geosyst., 6, Q03012, doi:10.1029/2004GC000821.

Ida, Y. (1972), Cohesive force across the tip of a longitudinal shear crack and Griffith's specific surface energy, J. Geophys. Res., 77, 3796-3805.

Li, Y.-G., P. Leary, K. Aki, and P. Malin (1990), Seismic trapped modes in the Oroville and San Andreas fault zones, Science, 249(4970), 763-766.

Linker, M. F., and J. H. Dieterich (1992), Effects of variable normal stress on rock friction: Observations and constitutive equations, J. Geophys. Res., 97, 4923-4940.

Lockner, D. A., and N. M. Beeler (2003), Stress-induced anisotropic poroelasticity response in sandstone, in Electronic Proceedings of the 16th ASCE Engineering Mechanics Conference, Seattle, WA. (http://www.ce. washington.edu/em03/proceedings/)

Lockner, D. A., and S. A. Stanchits (2002), Undrained poroelastic response of sandstones to deviatoric stress change, J. Geophys. Res., 107(B12), 2353, doi:10.1029/2001JB001460.

Lockner, D., H. Naka, H. Tanaka, H. Ito, and R. Ikeda (2000), Permeability and strength of core samples from the Nojima fault of the 1995 Kobe earthquake, in Proceedings of the International Workshop on the Nojima Fault Core and Borehole Data Analysis, edited by H. Ito et al., pp. 147152, U.S. Geol. Surv. Open File Rep., 00-129.

McGuire, J., and Y. Ben-Zion (2005), High-resolution imaging of the Bear Valley section of the San Andreas Fault at seismogenic depths with faultzone head waves and relocated seismicity, Geophys. J. Int., 163, 152164, doi:10.1111/j.1365-246X.2005.02703.x.

Noda, H., and T. Shimamoto (2005), Thermal pressurization and slip-weakening distance of a fault: An example of the Hanore fault, southwest Japan, Bull. Seismol. Soc. Am., 95(4), 1224-1233, doi:10.1785/0120040089.

O'Connell, R. J., and B. Budiansky (1974), Seismic velocities in dry and saturated cracked solids, J. Geophys. Res., 79, 5412-5426.

Poliakov, A. N. B., R. Dmowska, and J. R. Rice (2002), Dynamic shear rupture interactions with fault bends and off-axis secondary faulting, J. Geophys. Res., 107(B11), 2295, doi:10.1029/2001JB000572.

Prakash, V. (1998), Frictional response of sliding interfaces subjected to time varying normal pressures, J. Tribol., 120, 97-102.

Prakash, V., and R. J. Clifton (1993), Time resolved dynamic friction measurements in pressure-shear, in Experimental Techniques in the Dynamics of Deformable Solids, edited by A. K. T. Ramesh, pp. 33-48, Appl. Mech. Div., Am. Soc. of Mechanical Eng., New York.

Ranjith, K., and J. R. Rice (2001), Slip dynamics at an interface between dissimilar materials, J. Mech. Phys. Solids, 49, 341-361.

Renardy, M. (1992), Ill-posedness at the boundary for elastic solids sliding under Coulomb friction, J. Elastoplast., 27(3), 281-287, doi:10.1007/ BF00041691.

Rice, J. R. (2006), Heating and weakening of faults during earthquake slip, J. Geophys. Res., 111, B05311, doi:10.1029/2005JB004006.
Rice, J. R., and M. P. Cleary (1976), Some basic stress diffusion solutions for fluid-saturated elastic porous media with compressible constituents, Rev. Geophys. Space Phys., 14(2), 227-241.

Rice, J. R., C. G. Sammis, and R. Parsons (2005), Off-fault secondary failure induced by a dynamic slip-pulse, Bull. Seismol. Soc. Am., 95(1) 109-134, doi:10.1785/0120030166.

Richardson, E., and C. Marone (1999), Effects of normal stress vibrations on frictional healing, J. Geophys. Res., 104, 28,859-28,878.

Rubin, A. M., and J.-P. Ampuero (2007), Aftershock asymmetry on a bimaterial interface, J. Geophys. Res., 112, B05307, doi:10.1029/ 2006JB004337.

Rudnicki, J. W., and D. A. Koutsibelas (1991), Steady propagation of plane strain shear cracks on an impermeable plane in an elastic diffusive solid, Int. J. Solids Struct., 27, 205-225.

Rudnicki, J. W., and J. R. Rice (2006), Effective normal stress alteration due to pore pressure changes induced by dynamic slip propagation on a plane between dissimilar materials, J. Geophys. Res., 111, B10308, doi:10.1029/2006JB004396.

Tanimoto, T., and K. P. Sheldrake (2002), Three-dimensional S-wave velocity structure in Southern California, Geophys. Res. Lett., 29(8), 1223, doi:10.1029/2001GL013486.

Uenishi, K., and J. R. Rice (2003), Universal nucleation length for slipweakening rupture instability under nonuniform fault loading, J. Geophys. Res., 108(B1), 2042, doi:10.1029/2001JB001681.

Wang, H. F. (2000), Theory of Linear Poroelasticity with Applications to Geomechanics and Hydrogeology, $287 \mathrm{pp}$., Princeton Univ. Press, Princeton, N. J.

Weertman, J. (1963), Dislocations moving uniformly on the interface between isotropic media of different elastic properties, J. Mech. Phys. Solids, 11, 197-204.

Weertman, J. (1980), Unstable slippage across a fault that separates elastic media of different elastic constants, J. Geophys. Res., 85, 1455-1461.

Wibberley, C. A. J. (2002), Hydraulic diffusivity of fault gouge zones and implications for thermal pressurization during seismic slip, Earth Planets Space, 54(11), 1153-1171.

Wibberley, C. A. J., and T. Shimamoto (2003), Internal structure and permeability of major strike-slip fault zones: The Median Tectonic Line in Mie Prefecture, southwest Japan, J. Struct. Geol., 25(1), 59-78, doi:10.1016/S0191-8141(02)00014-7.

Xia, K., A. J. Rosakis, H. Kanamori, and J. R. Rice (2005), Laboratory earthquakes along inhomogeneous faults: Directionality and supershear, Science, 308(5722), 681-684, doi:10.1126/science.1108193.

E. M. Dunham and J. R. Rice, Department of Earth and Planetary Sciences and School of Engineering and Applied Sciences, Harvard University, 29 Oxford Street, Cambridge, MA 02138, USA. (edunham@ fas.harvard.edu; rice@esag.harvard.edu) 